\title{
VGluT2 Expression in Dopamine Neurons Contributes to Postlesional Striatal Reinnervation
}

\author{
Willemieke M. Kouwenhoven, ${ }^{1}$ Guillaume Fortin, ${ }^{1}$ Anna-Maija Penttinen, ${ }^{1}$ Clélia Florence, ${ }^{1}$ \\ Benoît Delignat-Lavaud, ${ }^{1}$ Marie-Josée Bourque, ${ }^{1}{ }^{\circledR}$ Thorsten Trimbuch, ${ }^{2}$ Milagros Pereira Luppi, ${ }^{3}$ \\ Alix Salvail-Lacoste, ${ }^{4}$ Pascale Legault, ${ }^{4}$ Jean-François Poulin, ${ }^{3}{ }^{\circledR}$ Christian Rosenmund, ${ }^{2}$ Raj Awatramani, ${ }^{3}$ and \\ ${ }^{-1}$ Louis-Éric Trudeau ${ }^{1}$ \\ ${ }^{1}$ Department of Pharmacology and Physiology, Department of Neurosciences, Groupe de Recherche sur le Système Nerveux Central, Faculty of \\ Medicine, Université de Montréal, Montréal, Québec H3C 3J7, Canada, ${ }^{2}$ Institut für Neurophysiologie, Charite Universitaetsmedizin, 10117 Berlin, \\ Germany, ${ }^{3}$ Department of Neurology and the Center for Genetic Medicine, Northwestern University, Chicago, Illinois 60611, and ${ }^{4}$ Department of \\ Biochemistry and Molecular Medicine, Faculty of Medicine, Université de Montréal, Montréal, Québec H3C 3J7, Canada
}

A subset of adult ventral tegmental area dopamine (DA) neurons expresses vesicular glutamate transporter 2 (VGluT2) and releases glutamate as a second neurotransmitter in the striatum, while only few adult substantia nigra DA neurons have this capacity. Recent work showed that cellular stress created by neurotoxins such as MPTP and 6-hydroxydopamine can upregulate VGluT2 in surviving DA neurons, suggesting the possibility of a role in cell survival, although a high level of overexpression could be toxic to DA neurons. Here we examined the level of VGluT2 upregulation in response to neurotoxins and its impact on postlesional plasticity. We first took advantage of an in vitro neurotoxin model of Parkinson's disease and found that this caused an average 2.5 -fold enhancement of Vglut2 mRNA in DA neurons. This could represent a reactivation of a developmental phenotype because using an intersectional genetic lineage-mapping approach, we find that $>\mathbf{9 8} \%$ of DA neurons have a VGluT2 ${ }^{+}$lineage. Expression of VGluT2 was detectable in most DA neurons at embryonic day 11.5 and was localized in developing axons. Finally, compatible with the possibility that enhanced VGluT2 expression in DA neurons promotes axonal outgrowth and reinnervation in the postlesional brain, we observed that DA neurons in female and male mice in which VGluT2 was conditionally removed established fewer striatal connections 7 weeks after a neurotoxin lesion. Thus, we propose here that the developmental expression of VGluT2 in DA neurons can be reactivated at postnatal stages, contributing to postlesional plasticity of dopaminergic axons.

Key words: axonal; dopamine; glutamate; Parkinson's; reinnervation; sprouting

Significance Statement

A small subset of dopamine neurons in the adult, healthy brain expresses vesicular glutamate transporter 2 (VGluT2) and thus releases glutamate as a second neurotransmitter in the striatum. This neurochemical phenotype appears to be plastic as exposure to neurotoxins, such as 6-OHDA or MPTP, that model certain aspects of Parkinson's disease pathophysiology, boosts VGluT2 expression in surviving dopamine neurons. Here we show that this enhanced VGluT2 expression in dopamine neurons drives axonal outgrowth and contributes to dopamine neuron axonal plasticity in the postlesional brain. A better understanding of the neurochemical changes that occur during the progression of Parkinson's disease pathology will aid the development of novel therapeutic strategies for this disease.

Received Apr. 8, 2020; revised Aug. 14, 2020; accepted Sep. 7, 2020.

Author contributions: W.M.K. and L.-E.T. designed research; W.M.K., G.F., A.-M.P., C.F., B.D.-L., M.-J.B., T.T., M.P.L., A.S.-L., J.-F.P., R.A., and C.R. performed research; W.M.K., G.F., A.-M.P., B.D.-L., P.L., and J.-F.P. analyzed data; W.M.K. and L.-E.T. wrote the paper.

This work was supported by the Canadian Institutes of Health Research (Grant MOP106556; to L.-E.T.); by pilot project grants from Parkinson Canada (to A.S.-L., P.L., and L.-E.T.); by the Brain Canada and Krembil foundations (to L.-E.T.); and by National Institutes of Health Grants R01-MH-110556, R01-NS-096240, and P50-DA-044121-01A1 (to R.A.). We thank Dr. Alexander Weil for access to the Stereolnvestigator stereological analysis workstation, Dr. Daniel Lévesque for access to his laboratory space and cryostat, and Dr. Sebastien
Talbot for access to his Nikon Ti2 epifluorescence microscope. We also thank Dominic Thibault for technical assistance with the viral preparations.

The authors declare no competing financial interests.

J.-F. Poulin's present address: Department of Neurology and Neurosurgery, McGill University, Montreal Neurological Institute, Montréal, Québec H3A 2B4, Canada.

Correspondence should be addressed to Louis-Eric Trudeau at louis-eric.trudeau@umontreal.ca.

https://doi.org/10.1523/JNEUROSCI.0823-20.2020

Copyright $\odot 2020$ the authors 


\section{Introduction}

A significant subset of adult ventral tegmental area (VTA) dopamine (DA) neurons expresses VGluT2, a vesicular glutamate transporter, and releases glutamate as a second neurotransmitter in the striatum, while only very few adult substantia nigra (SN) DA neurons have this capacity (Mendez et al., 2008; BérubéCarrière et al., 2012; Yamaguchi et al., 2013, 2015; Poulin et al., 2018). Adult VTA DA neurons that coexpress VGluT2 project to the medial shell of the nucleus accumbens, while the few adult $\mathrm{SN} \mathrm{DA} \mathrm{VGluT}^{+}{ }^{+}$neurons project densely to the tail of the striatum and more sparsely in rostral regions (Poulin et al., 2018). Interestingly, the level of Vglut2 transcript in adult DA neurons is very low (i.e., $10-15$ copies/cell), which is $\sim 10$-fold lower than the number of copies of the pivotal dopaminergic enzyme tyrosine hydroxylase (Th) mRNA in the same cells (Li et al., 2013). Based on qualitative in situ hybridization experiments (Yamaguchi et al., 2007), it appears that this level of Vglut2 mRNA is much lower compared with neurons that are classically considered to be "pure" glutamatergic neurons. The exact role of VGluT2 in DA neurons, and why it is expressed at this relatively low level, is currently unclear.

One hypothesis is that the low level of VGluT2 found in adult DA neurons represents a dormant, residual expression from early developmental stages and that VGluT2 expression can be reactivated in adulthood in response to cellular stress. Compatible with this hypothesis, gain-of-function and loss-offunction studies suggested that glutamate and VGluT2 contribute to axonal outgrowth by DA neurons (Schmitz et al., 2009; Fortin et al., 2012) and cell survival (Dal Bo et al., 2008; Shen et al., 2018; Steinkellner et al., 2018). Moreover, the NMDA receptor subunit NR1 is present on the axonal growth cone of cultured DA neurons, and DA neurons grown in the presence of a NR1 agonist display enhanced axonal growth, suggesting that an autocrine glutamate feedback loop may contribute to this axonal outgrowth (Schmitz et al., 2009).

VGluT2 in DA neurons also appears to contribute to a facilitation of DA release in ventral striatal regions. Decreased levels of evoked DA release in the nucleus accumbens were detected by fastscanning cyclic voltammetry in conditional VGluT2 knock-out (KO) mice (Hnasko et al., 2010; Fortin et al., 2012). Moreover, it has been proposed that the presence of VGluT2 on a synaptic vesicle promotes DA synaptic vesicle loading through altering the vesicular pH (Aguilar et al., 2017). However, several studies have shown that VGluT2 is typically segregated from TH, DA transporter (DAT), and VMAT2 in dopaminergic axonal varicosities (Fortin et al., 2012, 2019; Zhang et al., 2015; Silm et al., 2019).

Finally, VGluT2 is thought to contribute to DA neuron survival. Two weeks after either a 6-hydroxydopamine (6-OHDA) or MPTP lesion, more surviving DA neurons express Vglut2 (Dal Bo et al., 2008; Shen et al., 2018; Steinkellner et al., 2018). In addition, DA neurons lacking VGluT2 are more vulnerable to the neurotoxins 6-OHDA or MPTP. The hypothesis that VGluT2 contributes to the survival of DA neurons has previously been tested by viral overexpression of VGluT2 in DA neurons in an in vivo mouse model, leading to the following contrasting results: while one group reported enhanced survival of DA neurons in the MPTP model following VGluT2 overexpression (Shen et al., 2018), the other reported a massive cell loss caused by VGluT2 overexpression alone (Steinkellner et al., 2018).

In the current study, we aimed to clarify the consequences of the upregulation of VGluT2 in DA neurons in the postlesional brain. We show that in vitro 1-methyl-4-phenylpyridinium $\left(\mathrm{MPP}^{+}\right)$treatment more than doubles the initially low expression of Vglut2 in DA neurons. Furthermore, we reveal that moderate overexpression of VGLUT2 in cultured DA neurons increases axonal arborization, while constitutive removal of VGluT2 results in a regional DA striatal innervation deficit. Finally, we provide novel evidence that enhanced VGlutT2 in DA neurons after partial lesions contributes to reinnervation of the striatum.

\section{Materials and Methods}

\section{Animals}

The experimental protocols were approved by the Animal Handling and Ethics Committee of the Université de Montréal. Housing was at a constant temperature $\left(21^{\circ} \mathrm{C}\right)$ and humidity $(60 \%)$, under a fixed $12 \mathrm{~h} \mathrm{light/}$ dark cycle and free access to food and water.

\section{TH-GFP mice}

The TH-green fluorescent protein (GFP) transgenic mouse line $\mathrm{TH}$ EGFP/21-31, which carries the enhanced GFP gene under control of the TH promoter (Matsushita et al., 2002), was maintained on a C57BL6 background.

\section{Dat-Ires-Cre;AI9}

Dat-Ires-Cre animals (catalog \#006660, The Jackson Laboratory; Bäckman et al., 2006) were crossed with Ai9/tdTomato mice (catalog $\# 007905$, The Jackson Laboratory), allowing conditional expression of the red fluorescent protein tdTomato in DA neurons.

\section{Conditional VGluT2 knock-out mice}

6-OHDA and retrobead injection experiments were performed on mixedsex groups of conditional VGluT2 knock-out mice (VGluT2cKO) and control littermates (VGluT2cWT). Dat-Ires-Cre animals (catalog \#006660, The Jackson Laboratory) were crossed with Vglut $2^{\text {flox/flox }}$ mice (129/C57BL/6 mixed background) carrying the exon 2 surrounded by loxP site. A breeding colony was maintained by mating Dat-Ires-Cre;Vglut $2^{\text {flox/+ }}$ mice with $V g l u t 2^{\text {flox/flox }}$ mice. Twenty-five percent of the offspring from such mating were thus used as controls (i.e., Dat-Ires-Cre; $V$ glut $2^{\text {flox } /+}$ mice), and $25 \%$ lacked Vglut2 in DA neurons (i.e., Dat-Ires-Cre;Vglut $2^{\text {flox/flox }}$ mice).

\section{Constitutive VGluT2 knock-out mice}

The VGluT2 constitutive knock-out mouse line was previously validated by showing the absence of VGluT2 protein from whole brains obtained from VGluT2KO embryos at embryonic day 18.5 (E18.5; Moechars et al., 2006). Mice heterologous for VGluT2 (maintained on a C57BL/6 background) were crossed to generate litters that included wild-type (WT), heterozygous, and homozygous animals for the Vglut2 gene. The deletion of VGluT2 was previously validated, and genotyping was determined using specific primers (Moechars et al., 2006).

\section{Intersectional genetic mice}

Lineage fate-mapping experiments were performed using an intersectional strategy in which mice expressed VGluT2-Cre and TH-Flpo drivers in combination with the AI65 mouse line, allowing Flpo and Cre-dependent conditional expression of tdTomato; including the appropriate controls (either in VGluT2Cre ${ }^{+}$;ThFlp-;ttTomato or VGluT2Cre-; $\mathrm{ThFlp}^{+}$;tdTomato configuration). More details on these mice and breeding strategies were published previously (Poulin et al., 2018).

\section{Fluorescence-activated cell sorting-purified mesencephalic cultures} E11.5 cultures

$\mathrm{TH}-\mathrm{GFP}^{+}$, mix-sexed embryos were isolated at E11.5, considering the morning of detection of the vaginal plug as E0.5. The mesencephalon was dissected, and cells were prepared into a single-cell suspension using a previously published protocol (Fasano et al., 2008). Cells were purified by fluorescence-activated cell sorting (FACS) using a BD FACSAria (BD BioSciences) and seeded at a 10,000 cells/ml concentration.

\section{Postnatal cultures}

TH-GFP. The mesencephalon was dissected, and cells were taken from TH-GFP, mixed-sex postnatal day 1 (P1) to P2 mice, which were 
cryoanesthetized and decapitated for tissue collection to create a singlecell suspension using a previously published protocol (Fasano et al., 2008). Cells were purified by FACS before seeding at 10,000 cells $/ \mathrm{ml}$. Cells were treated with $2 \mu \mathrm{M} \mathrm{MPP}{ }^{+}$at $11 \mathrm{~d}$ in vitro (DIV11) and picked by a glass pipette for analysis at DIV14. Cells were treated with $10 \mu \mathrm{M}$ GDNF at DIV14 and picked by a glass pipette for analysis $3 \mathrm{~h}$ after treatment.

Dat-ires-cre;AI9. The mesencephalon was dissected, and cells taken from mixed-sex animals were dissociated to a single-cell suspension using a previously published protocol (Fasano et al., 2008). Cells were seeded at maximum concentration in $5 \mathrm{ml}$ of medium in a $25 \mathrm{~cm}^{2}$ Corning flask. Cells were treated with $2 \mu \mathrm{M} \mathrm{MPP}{ }^{+}$at DIV11 and purified by FACS at DIV14.

VGluT2-Venus overexpression

VGluT2 fused to Venus and Venus lentiviral vectors, using the Synapsin 1 promotor, were used for the overexpression of VGluT2 in primary mesencephalic neurons. The vectors were a gift from Etienne Herzog (University of Bordeaux, Bordeaux, France) and were produced according to the strategy previously published (Herzog et al., 2011). Neurons were infected $3 \mathrm{~h}$ after seeding [51,316-106,737 transducing units (TU)/

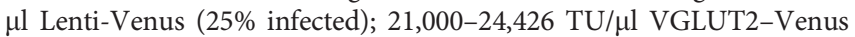
( $42 \%$ infected)].

\section{Immunohistochemistry \\ Embryos}

Coronal sections $(12 \mu \mathrm{m})$ were cut on a cryostat and collected on Superfrost Plus microscope slides, after which they were subsequently washed with PBS, permeabilized, blocked, and incubated overnight at $4^{\circ}$ C with a rabbit anti-TH antibody (1:1000; catalog \#AB152, Millipore Sigma) or rabbit anti-VMAT2 (1:2000; provided by G.W. Miller, Mailman School of Public Health, Columbia University; Cliburn et al., 2017). Slices were washed the following morning and incubated for a minimum of $2 \mathrm{~h}$ at room temperature (RT) with a secondary antibody (1:1000; rabbit Alexa Fluor-546 or rabbit Alexa Fluor-647). Finally, sections were washed, counterstained with DAPI, and embedded with Fluoromount-G (Southern Biotech).

\section{Neonates (P1 pups)}

Coronal sections $(25 \mu \mathrm{m})$ were cut on a cryostat, washed with PBS, permeabilized, blocked, and incubated overnight at $4^{\circ} \mathrm{C}$ with primary antiTH antibody (1:500; catalog \# P60101, Pel-Freez Biologicals). Slices were washed the following morning and incubated for a minimum of $2 \mathrm{~h}$ at RT with secondary antibody (rabbit Alexa Fluor-488 and rabbit Alexa Fluor-647, Thermo Fisher Scientific). Finally, sections were washed, counterstained with DAPI, and mounted on microscope slides.

\section{Adult brain slices}

Coronal sections $(40 \mu \mathrm{m})$ were cut on a cryostat and stored in antifreeze solution at $-20^{\circ} \mathrm{C}$ until used. Slices were washed with PBS, permeabilized, blocked, and incubated overnight at $4^{\circ} \mathrm{C}$ with rabbit anti- $\mathrm{TH}$ antibody (1:1000; AB152, Millipore Sigma). Slices were washed the following morning and incubated for a minimum of $2 \mathrm{~h}$ at RT with secondary antibody (1:1000; ant-rabbit Alexa Fluor-647, Thermo Fisher Scientific). Finally, sections were washed, counterstained with DAPI, and mounted with Fluoromount-G (Southern Biotech) on Superfrost Plus microscope slides.

\section{Immunocytochemistry on cell cultures}

Cultures were fixed with $4 \%$ paraformaldehyde (PFA) and permeabilized, and nonspecific binding sites were blocked. To study the overexpression/expression of VGluT2 protein by postnatal DA neurons, a chicken anti-GFP (1:2000; catalog \#GFP-1020, Aves Labs) and a rabbit anti-VGluT2 (1:2000; catalog \#135402, Synaptic Systems). To study the expression of VGluT2 by embryonic DA neurons a mouse anti-VGluT2 (1:4000; catalog \#AB5504, Millipore) and primary rabbit anti-TH antibody (1:1000; catalog \#AB152, Millipore Sigma) were used. Cultures were washed the following morning and incubated for a minimum of 2 $\mathrm{h}$ at RT with secondary antibodies (anti-rabbit Alexa Fluor-546/antichicken Alexa Fluor-488 and rabbit Alexa Fluor-546/anti-mouse Alexa
Fluor-647/anti-chicken Alexa Fluor-488, respectively; all 1:1000; Thermo Fisher Scientific). Finally, coverslips were washed, counterstained with DAPI, and mounted in Fluoromount-G (Southern Biotech) on Superfrost Plus microscope slides.

\section{Image acquisition with confocal microscopy}

All in vitro images were acquired with a FluoView FV1000 confocal microscope system (Olympus Canada). For the quantification of VGluT2 protein in postnatal cells, 10 random field of views were chosen per coverslip, and images were acquired using a $546 \mathrm{~nm}$ excitation laser and a $10 \times$ water-immersion objective. For the analysis of the length of neuronal processes, images of five isolated, $\mathrm{TH}^{+}$neurons per coverslip were acquired. For quantification of TH and VMAT2 immunoreactivity in E18.5 dorsal and ventral striatum from VGluT2WT and VGluT2KO animals, two fields of view per hemisphere were acquired with a $60 \times$ oilimmersion objective. Three to four animals of each genotype were analyzed.

\section{Quantitative analysis of mesencephalon at E18.5}

Images were taken with a Nikon Ti2 epifluorescence microscope, and, using ImageJ, color combined images were split into single-channel images and subsequently converted into binary images using default settings, after which the binary DAPI image was used as an overlay on top of the GFP image using image calculator. This created a binary image of only the cells that were positive for both DAPI and TH (cellular particles binary image), which were then counted using the "analyze particles" function of ImageJ.

\section{Double fluorescence in situ hybridization}

TH-GFP ${ }^{-}$embryos were isolated at E11.5 and E14.5 and immediately frozen on dry ice. Sagittal slices of $10 \mu \mathrm{m}$ were collected on Superfrost Plus slides. Double fluorescence in situ hybridization (FISH) was performed as described previously (Fasano et al., 2017; Gras et al., 2008). Briefly, sections were fixed with $4 \%$ PFA (Tousimis) for $10 \mathrm{~min}$, washed with PBS, and acetylated with acetic anhydride for $10 \mathrm{~min}$. Prehybridization was conducted by incubating the slides with hybridization mix (50\% Formamide, SigmaAldrich; SSC, Thermo Fisher Scientific; Escherichia coli tRNA, Roche; Denhardt's Solution, Sigma-Aldrich; Salmon Sperm DNA, Sigma-Aldrich) for $30 \mathrm{~min}$ at RT in a wet incubation chamber. Hybridization occurred overnight at $60^{\circ} \mathrm{C}$ in a wet incubation chamber. After hybridization, slides were washed using several steps of SSC washes $(2 \times$ SSC, $0.2 \times$ SSC) and incubated with blocking solution (FBS heat-inactivated blocking reagent, Roche). Probes were detected using a $1 \mathrm{~h}$ incubation at RT with anti-fluorescein-POD (1:2500; Peroxidase, Roche) and anti-digoxigenin (DIG)POD antibodies (1:2500; Roche). Sections were passed through several steps of washes in maleic acid buffer and in PBS-Tween (Thermo Fisher Scientific), after which the DIG probes were visualized using tyramide signal amplification (TSA)-Cy3 (PerkinElmer) and fluorescein probes were visualized using TSA-Biotin (PerkinElmer) and Neutravidin-Oregon Green (Thermo Fisher Scientific). All sections were counterstained using DAPI and mounted using Fluoromount. Double FISH was performed with RNA probes labeled with DIG (Vglut2) and RNA probes labeled with Fluorescein (Th). The Th probe was a gift from Marten Smidt (University of Amsterdam, Amsterdam, The Netherlands), and their sequences have been previously published (Grima et al., 1985; Jacobs et al., 2007). The sequence of the Vglut2 probe was published before (Herzog et al., 2001), and the probe was provided by Salah El Mestikawy (McGill University, Montreal, QC, Canada).

\section{Single-cell and bulk real-time quantitative PCR}

Single-cell real-time quantitative PCR

Experimental design was based on a previously described protocol (Fortin et al., 2019). In short, cultures were treated with $2 \mu \mathrm{M} \mathrm{MPP}{ }^{+}$at DIV11 and TH-GFP ${ }^{+}$cells were collected from coverslips using a glass pipette at DIV14 and placed directly in an aliquot containing $0.5 \mathrm{ml}$ of RNase Out (Thermo Fisher Scientific) and $0.5 \mathrm{ml}$ of DTT (Thermo Fisher Scientific). Then, total RNA from each cell was reverse transcribed in a total of $20 \mu \mathrm{l}$. Quantitative PCR (qPCR) was performed using SYBR Green PCR Master Mix (Quantabio), on a LightCycler 96 
system (Hoffmann-La Roche). The presence of a cell in each sample was confirmed by detecting the presence of glyceraldehyde-3-phosphate dehydrogenase (Gapdh) mRNA. Calculation of the absolute copy number for Th and VgluT2 in each cell was determined with an external calibration curve based on a Th or VGluT2 plasmid cDNA (obtained, respectively, from Sino Biological and Harvard University, Cambridge, MA).

\section{Bulk real-time qPCR}

Cells were treated with $2 \mu \mathrm{M} \mathrm{MPP}{ }^{+}$at DIV11 and purified by FACS at DIV14. Then, total RNA was isolated from harvested cells with TRIzol (Thermo Fisher Scientific) according to manufacturer instructions and was reverse transcribed in a total of $20 \mu \mathrm{l} / \mu \mathrm{g}$ RNA. Quantitative PCR was performed using SYBR Green PCR Master Mix (Quantabio) on a Light Cycler 96 system (Hoffmann-La Roche). For normalization of mRNA expression levels, the Gapdh was used as a reference gene; qPCR results were analyzed using the $\Delta \Delta \mathrm{Cq}$ method.

Primers were synthesized by AlphaDNA. Primers for qPCR were as follows: Th, 5' ${ }^{\prime}$-TGGCCTTCCGTGTGTTT- $3^{\prime}$ and $5^{\prime}$-AATGTCCTGG GAGAACTGG-3'; Vglut2, 5'-CCTTTTGTGGTTCCTATGCT-3' and 5'-GCTCTCTCCAATGCTCTCC-3'; and Gapdh, 5'-GGAGGAAACC TGCCAAGTATGA-3' and $5^{\prime}$-TGAAGTCGCAGGAGACAACC- $3^{\prime}$.

\section{Embryo collection and tissue preparation}

Embryos from several mouse lines were isolated at E11.5, E14.5, and E18.5, considering the morning of detection of the vaginal plug as E0.5. Embryos were isolated in ice-cold PBS and fixed in 4\% PFA for 24-48 h for immunohistochemistry or freshly frozen on dry ice for in situ hybridization. VGluT2WT and VGluT2KO embryos were cryoprotected and shipped from the laboratory in Berlin (Germany) to Montreal (Canada) in $30 \%$ sucrose in PBS, at room temperature. Upon arrival, embryos were stored immediately at $4^{\circ} \mathrm{C}$ and frozen on dry ice before cryostat sectioning $(12 \mu \mathrm{m})$.

\section{Surgeries and tissue preparation}

The 6-OHDA surgery protocol used here was previously described (Giguère et al., 2019). In short, VGluT2cWT and VGluT2cKO animals (2-6 months old) were anesthetized with isoflurane (Aerrane, Baxter) and fixed on a stereotaxic frame (Stoelting). Animals received a 6OHDA injection $[0.5 \mu \mathrm{l}$ of 6 -OHDA $(3 \mathrm{mg} / \mathrm{ml})$ in $0.2 \%$ ascorbic acid solution] in the dorsal striatum [bregma coordinates: anteroposterior (AP), $0.5 \mathrm{~mm}$; mediolateral (ML), $2.0 \mathrm{~mm}$; dorsoventral (DV), -3.0 $\mathrm{mm}]$. The norepinephrine transporter blocker desipramine $(2.5 \mathrm{mg} / \mathrm{ml}$; $10 \mu \mathrm{l} / \mathrm{g}$ ) was injected $45 \mathrm{~min}$ before 6-OHDA. Animals recovered in their own cages. After 6 weeks, animals received $100 \mathrm{nl}$ of red fluorescent retrobeads ( $\mathrm{Rb})$ IX (1:1 diluted in saline; Lumafluor) using the same surgery and anesthesia protocol and bregma coordinates. One week after the $\mathrm{Rb}$ injections, animals were anesthetized using pentobarbital $\mathrm{NaCl}$ saline solution $(7 \mathrm{mg} / \mathrm{ml})$ injected intraperitoneally, after which they were perfused with $50 \mathrm{ml}$ of PBS followed by $100 \mathrm{ml}$ of PFA $4 \%$ using an intracardiac needle at a rate of $25 \mathrm{ml} / \mathrm{min}$. Brains were postfixed in $4 \%$ PFA for $48 \mathrm{~h}$, transferred to $30 \%$ sucrose in PBS solution, and stored at $-80^{\circ} \mathrm{C}$ before being sectioned at the cryostat at $40 \mu \mathrm{m}$. Brains displaying unsuccessful lesions or mislocated retrobead injections (i.e., outside the striatal lesion zone) were excluded from analysis (see Fig. $5 A$, inset).

\section{Analysis of $\mathrm{Rb}^{+} / \mathrm{TH}^{+}$cells in mesencephalon}

The striatum was imaged using the Nikon Ti2 microscope using a $20 \times$ objective. Using Nikon Elements software, the circumference of Rb fluorescence at the injection site was traced to calculate the area. The number of counted $\mathrm{Rb}^{+}$cells in the mesencephalon of each mouse was divided by the area of $\mathrm{Rb}$ in the striatum to normalize for variation in the injection volume. Stacks of $5 \mu \mathrm{m}$ were acquired (5-6 slices/stack), taking a 2 $\times 2 \mathrm{~mm}$ field of view of the ipsilateral injection side, for TH (Alexa Fluor-647) and retrobeads (RFP). Stitched images were analyzed as maximum intensity projections. $\mathrm{TH}^{-}$and $\mathrm{TH}^{+}$cells containing $\mathrm{Rbs}$ were counted blindly, manually.

\section{Analysis of TH immunoreactivity in dorsal striatal sections}

The striatal slices were imaged using a Nikon Eclipse TE200 epifluorescence inverted microscope using a $10 \times$ objective (numerical aperture 0.25 ) and Micro-Manager software and analyzed by a blinded observer. ImageJ was used to draw the circumference of the dorsal striatum, on both ipsilateral and contralateral sides and to determine the average THimmunoreactivity (IR) in each region of interest. The average TH-IR of each ipsilateral side was divided by the average TH-IR of the contralateral side. These values were added to obtain the total TH-IR ratio per brain to quantify the lesion size per brain.

\section{Stereology and DAB immunohistochemistry}

Brain sections $(40 \mu \mathrm{m})$ of VGluT2cWT and VGluT2cKO animals that had undergone surgery were washed with PBS, and the endogenous peroxidase activity was quenched with $0.9 \%$ hydrogen peroxidase. Slices were permeabilized and nonspecific binding sites were blocked before incubation with rabbit anti-TH antibody (1:1000; catalog \#AB152, Millipore Sigma) for $48 \mathrm{~h}$. Subsequently, slices were incubated at $4^{\circ} \mathrm{C}$ for $12 \mathrm{~h}$ with goat anti-rabbit biotin-SP-AffiniPure secondary antibody (1:200; catalog \#111-065-003, Jackson ImmunoResearch), and finally for $3 \mathrm{~h}$ with horseradish peroxidase streptavidin (catalog \#016-030-084, Cedarlane). The DAB reaction was conducted for $15 \mathrm{~s}$, then stopped by incubation with $0.1 \mathrm{M}$ acetate buffer and slices were mounted on Superfrost Plus microscope slides. They were left to dry for $96 \mathrm{~h}$, after which they were counterstained with Cresyl Violet and dehydrated, and, finally, slides were sealed with Permount Mounting Medium (catalog \#SP15-100, Thermo Fisher Scientific) using glass coverslips.

\section{Stereological counting}

SN TH-immunoreactive neurons were counted in every sixth section using a $100 \times$ oil-immersion objective on a Leica DMRE Stereomicroscope equipped with a motorized stage by a blinded observer. A $60 \times 60 \mu \mathrm{m}^{2}$ counting frame was used in the Stereo Investigator sampling software with (MBF Bioscience) a $20 \mu \mathrm{m}$ optical dissector ( $2 \mu \mathrm{m}$ guard zones) and counting site intervals of $100 \mu \mathrm{m}$ after a random start. Stereological estimates of the total number of TH-IR neurons within ipsilateral SN were obtained, and these numbers are presented relative to the number of $\mathrm{TH}^{+}$neurons counted on the intact contralateral side.

\section{Statistics}

Data are represented throughout as the mean \pm SEM. Statistically significant differences were analyzed with a Student's $t$ test, two-way ANOVA (Tukey's post hoc test), or a Fisher's exact test, as appropriate. A $p$ value $<0.05\left(^{\star}\right)$ was considered significant $\left({ }^{* *} p<0.01,{ }^{* *} p<0.001\right)$.

\section{Results}

\section{VGluT2 expression doubles in cultured DA neurons after exposure to the neurotoxin MPP ${ }^{+}$}

The expression level of Vglut2 in healthy, adult DA neurons is low, $\sim 10$ copies, compared with $\sim 100$ copies of $T h$ (Li et al., 2013). The reported proportion of VGluT2 ${ }^{+}$DA neurons in control animals varies between $7 \%$ and $20 \%$ (Table 1 ). This variability may well be caused by differences in age, anatomic location, and detection technique, and because these low levels of Vglut2 expression are difficult to detect (Table 1). A gradually emerging literature, however, suggests that neurotoxic lesions increase the likelihood of VGluT2 expression in surviving DA neurons (Dal Bo et al., 2008; Shen et al., 2018; Steinkellner et al., 2018). However, it is currently not clear whether this is caused by enhanced VGluT2 expression per DA neuron or enhanced survival of DA neurons that coexpress Vglut2 and Th. To answer this question and to quantify the magnitude of Vglut2 upregulation, we used $\mathrm{MPP}^{+}$to mimic Parkinson's disease (PD)-related cellular stress. We examined the possibility that $\mathrm{MPP}^{+}$triggers increased expression of Vglut2 in parallel to its well known ability to reduce Th expression (Bowenkamp et al., 1996). 
Table 1. Overview of reported prevalence of Vglut2 DA neurons

\begin{tabular}{llllll}
\hline Cells & Age & VGluT2 $^{+} / \mathrm{Th}^{+}$in control (\%) & VGluT2 $^{+} / \mathrm{Th}^{+}$postlesion (\%) & Technique & Article \\
\hline VTA, in vivo, rat & P15 & 7 & 26 & ISH & Dal Bo et al., 2008 \\
SN, in vivo, mouse & P556 & 9 & 21 & RNAscope & Steinkellner et al., 2018 \\
VTA, in vivo, mouse & $>$ P56 & 15 & 19 & RNAscope & Steinkellner et al., 2018 \\
SN, in vivo, mouse & P556 & 20 & 48 & RNAscope & Shen et al., 2018 \\
VTA, in vivo, mouse & $>$ P56 & 17 & 35 & RNAscope & Shen et al., 2018 \\
SN, in vitro, mouse & P0-P2 & 28 & 62 & Single-cell qPCR & 20-copy limit current manuscript \\
SN, in vitro, mouse & P0-P2 & 49 & 64 & Single-cell qPCR & $10-$ copy limit current manuscript \\
SN, in vivo, mouse & P29 & 21 & NA & Intersectional genetics & Chuhma et al., 2018 \\
\hline
\end{tabular}

To test this hypothesis, we treated cultured SN DA neurons, taken from mice expressing the red fluorescent reporter TdTomato in DA neurons (DatCre; AI9 neonatal pups) with a low dose $(2 \mu \mathrm{M})$ of $\mathrm{MPP}^{+}$. After $72 \mathrm{~h}$, we used FACS to purify tdTomato $^{+}$DA neurons and performed qPCR (Fig. 1A-C). Quantification of the number of FACS-purified neurons demonstrated no significant cell loss after the $2 \mu \mathrm{M} \mathrm{MPP}{ }^{+}$treatment, confirming the chosen dose to be sublethal ( $n=3$ cultures, Student's paired $t$ test, $p=0.39$; Fig. $1 B$ ). qPCR on RNA extracted from these purified samples confirmed a significant threefold decrease of $T h$ transcript level (Fig. 1C; Student's $t$ test, $p<0.0001 ; n=3$ cultures) and revealed a twofold increase of Vglut2 expression after $\mathrm{MPP}^{+}$treatment (Fig. $1 C$; Student's $t$ test, $p=0.02 ; n=3$ cultures).

To investigate whether all DA SN neurons possess the potential to upregulate Vglut2 expression, we repeated the $\mathrm{MPP}^{+}$paradigm in an independent set of experiments, in which we treated cultured SN DA neurons, taken from TH-GFP ${ }^{+}$neonatal pups, with $2 \mu \mathrm{M} \mathrm{MPP}{ }^{+}$for $72 \mathrm{~h}$ at DIV11. We collected single DA neurons using a glass pipette, after which we performed single-cell qPCR to determine the exact copy number of Th and Vglut2 present per SN DA neuron (Fig. $1 D-H)$. We confirmed that $\mathrm{MPP}^{+}$caused a significant decrease in Th transcript expression per SN neuron (Fig. $1 E$; 122 vs 31 copies, Student's $t$ test, $p=0.04$ ). Moreover, the proportion of $\mathrm{TH}-\mathrm{GFP}^{+} \mathrm{DA}$ neurons that still expressed Th above the threshold detection level (i.e., 10 mRNA copies) decreased significantly after the $\mathrm{MPP}^{+}$treatment [24 of 35 neurons $(69 \%)$ vs 10 of 34 neurons (30\%); Student's $t$ test, $p<0.01$; Fig. $1 F]$. To investigate the effect of $\mathrm{MPP}^{+}$treatment on the glutamatergic identity of SN DA neurons, we considered two possible thresholds on the minimal number of Vglut 2 mRNA copy numbers to consider a neuron as positive. We compared a threshold of 10 mRNA copies per DA neuron, based on adult SN DA Vglut2 expression (Li et al., 2013) with a more stringent 20-copy threshold. Using these parameters, we first observed that $\mathrm{GFP}^{+} \mathrm{SN}$ neurons displayed a more than twofold increase in Vglut2 mRNA copy number after $\mathrm{MPP}^{+}$treatment (Fig. 1G; 10-copy limit: 33 vs 81 copies, Student's $t$ test, $p=0.0009$; 20 -copy limit: 46 vs 85 copies, $p=0.03$ ). Next, we investigated whether the percentage of SN DA neurons that expressed Vglut2 changed after $\mathrm{MPP}^{+}$treatment. Using the 10 copies criterion, we observed that almost $50 \%$ of the control SN neurons were positive for Vglut2 (Fig. $1 H ; 17$ of 35 neurons). In comparison, using the 20 -copy threshold, only $28 \%$ of DA neurons were considered positive for Vglut2 (Fig. 1I). After $\mathrm{MPP}^{+}$ treatment, the percentage of DA neurons expressing $\geq 20$ copies of Vglut2 mRNA enhanced to $\sim 60 \%$ (Fig. 1I; Fisher's exact test, $p=0.007$ ), a conclusion that was masked when using 10 copies as the criterion. Together, these experiments suggest that a large proportion of postnatal SN DA neurons contain some level of Vglut2 mRNA and that a large subset of these neurons has the potential to more than double their expression of Vglut2 on exposure to cellular stress.

Nearly all DA neurons have a VGluT2 lineage and VGluT2 is ubiquitously expressed in DA neurons at E11.5

In line with our observation of the presence of Vglut 2 mRNA in a large subset of SN DA neurons, a recent study reported that most DA neurons possess a VGluT2 ${ }^{+}$lineage (Steinkellner et al., 2018). We next aimed to validate previously published lineage data taking advantage of an intersectional genetic approach (Poulin et al., 2018) using VGluT2Cre and Th Flpo as drivers to visualize only cells with a history of coexpression of $T h$ and Vglut2 by the expression of tdTomato (Fig. $2 A$ ). Using TH protein as a marker for DA neurons, we determined that almost all $\mathrm{SN}(99 \pm 0.3 \%, n=3)$ and VTA DA neurons $(98 \pm 0.3 \%, n=3)$ have a VGluT2 lineage (Fig. $2 B, C$ ). Interestingly, in these experiments we also noted the presence of a subpopulation of neurons with VGluT2 lineage that were $\mathrm{TH}^{-}$, suggesting that the dopaminergic phenotype in these neurons was downregulated below detection level (Fig. 2C, white arrow). Of note, no tdTomato expression was observed in $\mathrm{P} 1$ brains obtained from appropriate controls (VGluT2Cre ${ }^{+}$;ThFlp-;tdTomato or Vglut2Cre-;ThFlp ${ }^{+}$; tdTomato, $n=2$ each), confirming the absence of leakage and the validity of this approach (Fig. 2D). Together, these experiments indicate that practically all DA neurons have expressed VGluT2 before P1. These data thus confirm and complement recent findings (Steinkellner et al., 2018).

These data therefore show that all DA neurons must have had a history of VGluT2 expression before birth. To determine at what developmental stage Vglut2 is expressed in DA neurons, we used double fluorescence in situ hybridization and observed overlapping Vglut2 and Th expression patterns. We investigated the following two embryonic stages that represent a time point of importance during dopaminergic development: E11.5, the age at which DA progenitors start expressing Th and first develop axons; and E14.5, at which age DA neurons are considered terminally differentiated (Smidt and Burbach, 2007; Arenas et al., 2015). We observed overlapping Vglut2 and Th expression patterns at E11.5 in the mesencephalon (Fig. 2D). Moreover, to establish whether VGluT2 protein is also found in DA neurons at this age, we dissected the mesencephalon of E11.5 pups from TH-GFP animals and selected $\mathrm{TH}^{+}$cells using FACS. Consistent with the Vglut2 transcript data, we observed clear VGluT2 protein in TH-GFP ${ }^{+}$DA neurons that were grown for DIV1 (Fig. $2 E$ ). VGluT2 protein was located both in $\mathrm{TH}^{+}$cell bodies as well as in the growth cones of these developing neurons (Fig. 2E, insert). Interestingly, at E14.5 the transcript expression pattern of Vglut2 no longer overlapped completely with the expression of Th. Vglut2 transcript was still present in $\mathrm{Th}^{+}$cells located in medial sections of the mesencephalon but was no longer present in lateral sections (Fig. $2 F$ ), indicating that the downregulation of 
A
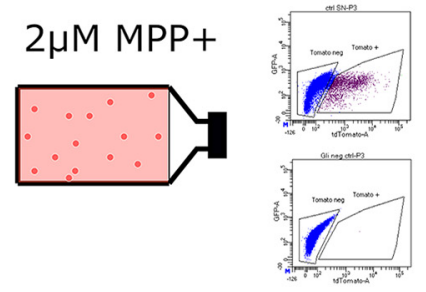

D

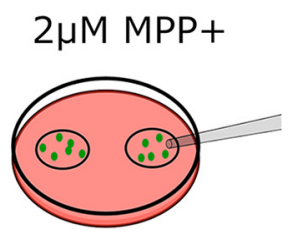

G

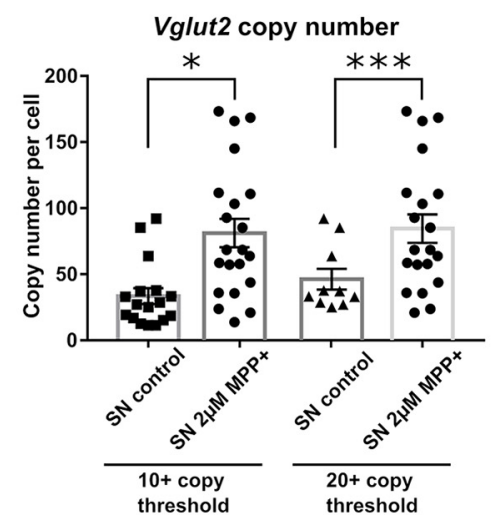

B

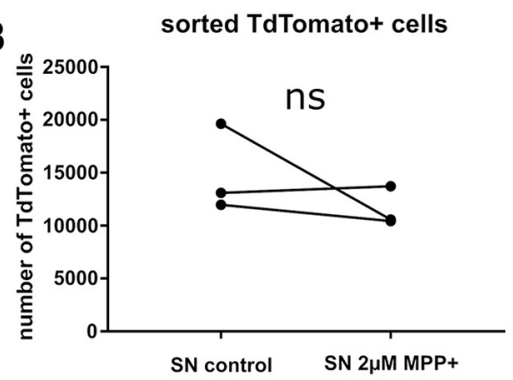

E

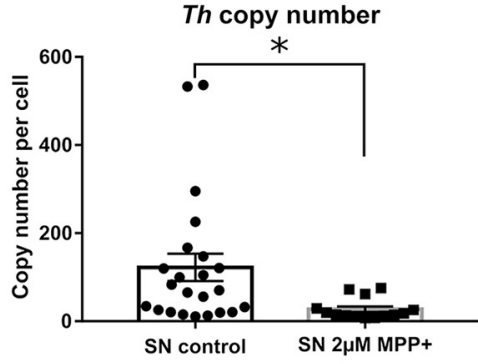

H

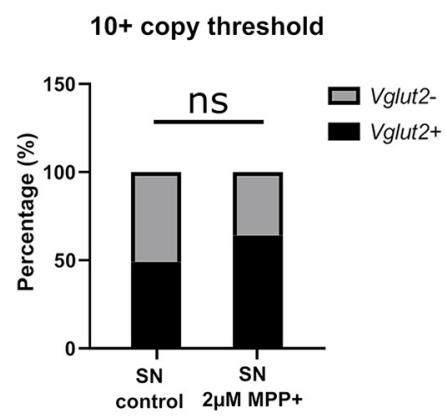

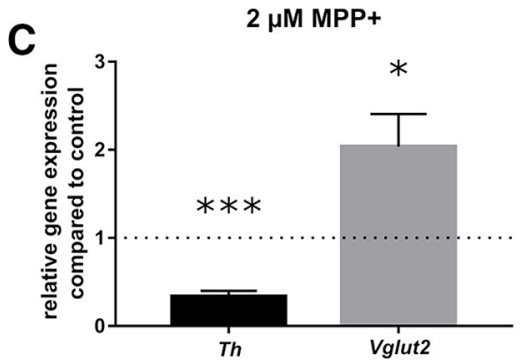

F

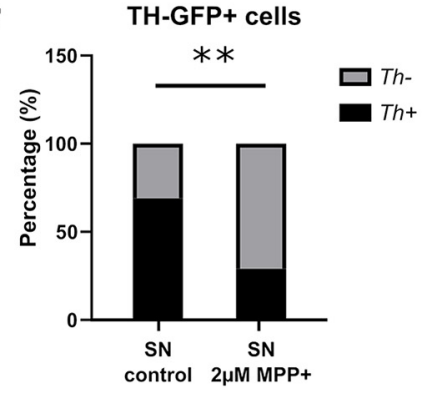

I

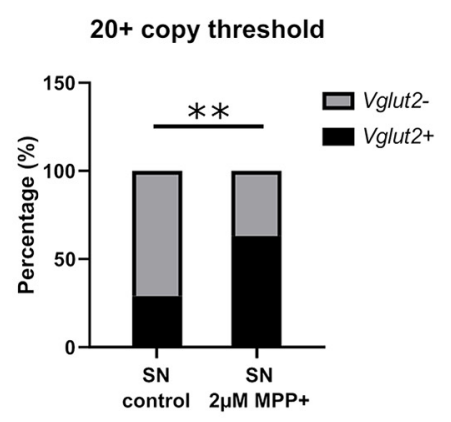

Figure 1. Vglut2 expression doubles in cultured DA neurons after exposure to the neurotoxin $\mathrm{MPP}^{+}$. $\boldsymbol{A}$, Schematic representation of the experimental setup used: Dat-Ires-Cre,tdTomato SN neurons were cultured in a $5 \mathrm{ml}$ flask, received $2 \mu \mathrm{m} \mathrm{MPP}{ }^{+}$at DIV11, and were purified using FACS at DIV14. B, No significant change in the number of tdTomato ${ }^{+}$neurons was observed between control and $\mathrm{MPP}^{+}$conditions (Student's paired $t$ test, $n=3$ cultures). C, A significant decrease of $T h$ transcript (Student's $t$ test, $p<0.001, n=3$ ) and a significant increase of Vglut2 expression was observed after $\mathrm{MPP}^{+}$treatment (Student's $t$ test, $p<0.05, n=3$ ). The dotted line represents untreated control expression. D, TH-GFP ${ }^{+}$SN neurons were grown in a Petri dish and were collected with a glass pipette at DIV14, after $72 \mathrm{~h}$ of $2 \mu \mathrm{MMPP}{ }^{+}$treatment. $\boldsymbol{E}$, SN neurons expressed on average $122( \pm 31, n=23)$ copies of $T h$ transcript at DIV14, and this decreased to $30( \pm 7, n=12)$ copies after MPP ${ }^{+}$treatment (Student's $t$ test, $p<0.05$ ). $\boldsymbol{F}$, Significantly fewer SN neurons expressed detectable levels of Th mRNA after MPP ${ }^{+}$treatment (12 of 33), compared with control (23 of 35, Fisher's exact test, $p<0.01$ ). G, SN neurons contained on average 33 ( \pm 6 copies, $n=17$ ) of Vglut2 transcript at DIV14, and this increased to $81( \pm 11, n=21)$ copies after MPP ${ }^{+}$treatment (Student's $t$ test, $p<0.001$ ). $\boldsymbol{H}$, I, Significantly more SN neurons expressed over 20 Vglut2 copies after MPP ${ }^{+}$treatment (20 of 32) compared with control (10 of 35, Fisher's exact test, $\left.p<0.01\right)$. Using the 10-copy limit, we observed the same trend, which was not statistically significant (17 of 35 Vglut2 SN neurons in control vs 21 of 33 after MPP ${ }^{+}$treatment). $\boldsymbol{H}_{,}{ }^{p} p<0.05,{ }^{* *} p<0.01,{ }^{* * *} p<0.001$, ns, no significant difference.

Vglut2 expression, which is observed in most adult DA neurons, is initiated during embryonic development. Our findings are compatible with a very recent report investigating early Vglut2 expression in dopaminergic nuclei during embryonic development, also showing ubiquitous Vglut2 expression in DA neurons at E11.5 and revealing that Vglut2 is expressed in the mesencephalon before the first DA markers such as Nurrl (Dumas and Wallén-Mackenzie, 2019).

TH downregulation in DA axons in dorsal caudal region of embryonic striatum in the absence of VGluT2

Knowing that VGluT2 is ubiquitously expressed by embryonic DA neurons, we wondered what role VGluT2 could play in DA neurons at such an early age. Previous loss-of-function studies using the DA transporter (Dat/Slc6a3) as a driver to conditionally delete VGluT2 in DA neurons revealed smaller axonal arborizations when postnatal DA neurons were grown in vitro; as well as a reduced number of $\mathrm{TH}^{+}$neurons in the adult brain (Hnasko et al., 2010; Fortin et al., 2012). Intriguingly, the latter finding was not replicated in Dat-Ires-Cre;Vglut2KO mice in which Dat levels were not decreased secondary to Cre-driver insertion in the Dat gene locus (Steinkellner et al., 2018). Here, aiming to better understand the role of VGluT2 during early embryonic development at a time point earlier than Dat gene (and thus Cre) activation at E15 (Bäckman et al., 2006), we investigated the DA system in constitutive VGluT2 knockout (VGluT2KO mice). We examined mice at E18.5 because these animals display perinatal mortality (Wallén-Mackenzie et al., 2006). Using TH immunohistochemistry, we observed no overt macroscopic changes in the organization of the DA system in VGluT2KO mice compared with VGluT2WT littermate controls (Fig. 3A). Furthermore, quantification of $\mathrm{TH}^{+}$neurons revealed 

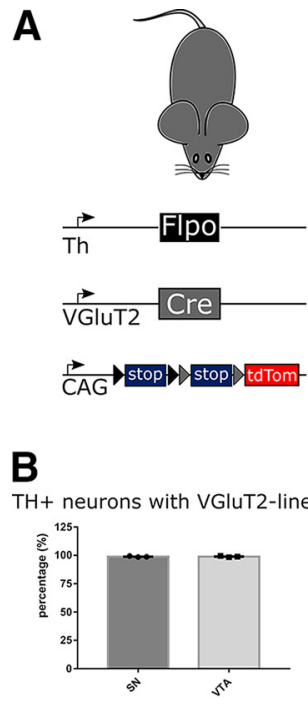

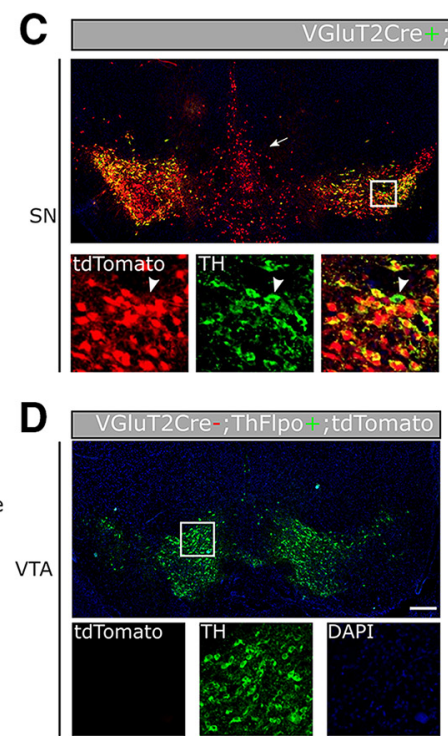

D

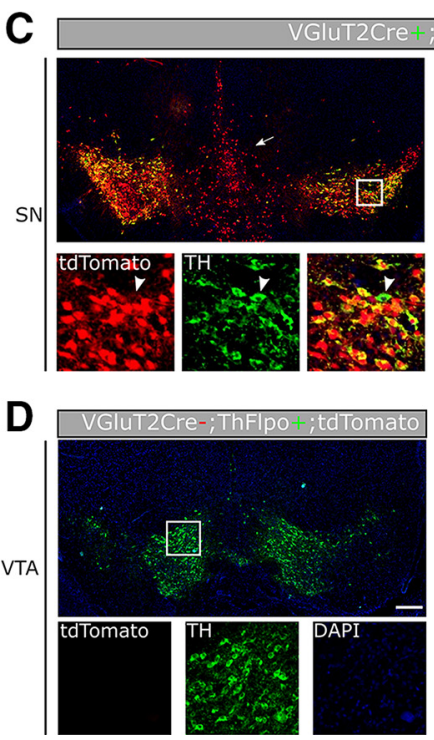

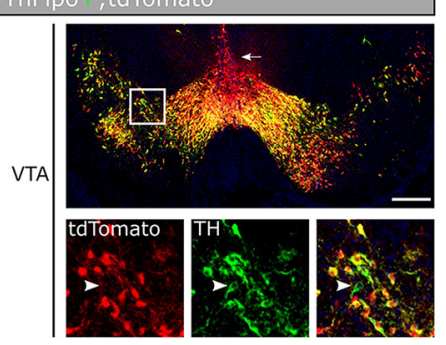

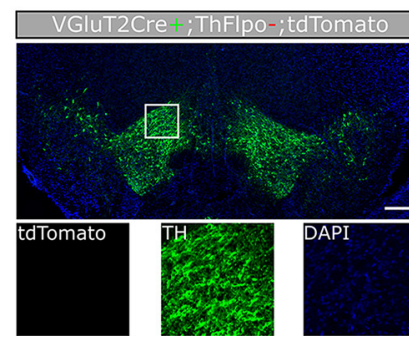

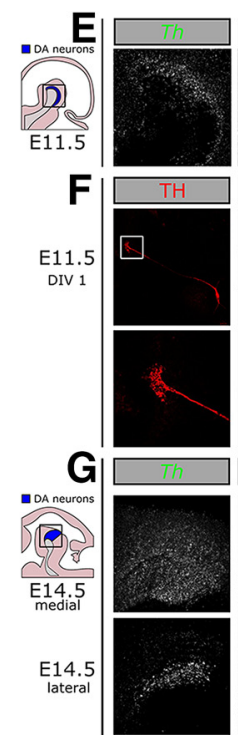
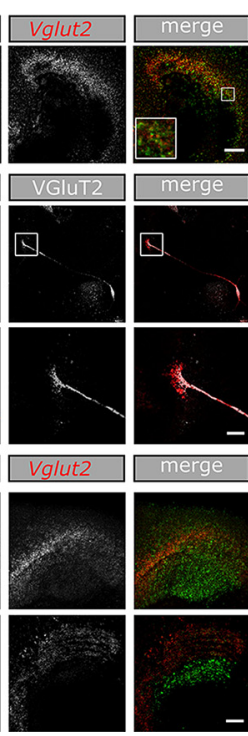

Figure 2. Most DA neurons have a Vglut2 expression history. A, Schematic representation of the intersectional genetic approach used the following: mice expressing a Th-Flpo and VGluT2Cre conditional construct will express tdTomato only in cells that have expressed both Th and Vglut2 genes. B, C, A total of $98 \%$ of SN and VTA TH ${ }^{+}$neurons colocalize with tdTomato. Only a

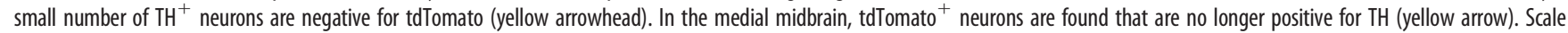
bar, $200 \mu \mathrm{m}$. D, Brains taken from P1 VGluT2Cre ${ }^{+}$;ThFlp-;tdTomato or VGluT2Cre-;ThFlp ${ }^{+}$;tdTomato pups showed no tdTomato expression. Scale bar, $200 \mu$ m. $\boldsymbol{E}$, Fluorescence in situ hybridization reveals that Vglut2 expression overlaps with that of $T h$ in the mesencephalon at E11.5. Scale bar, $100 \mu m$. $\boldsymbol{F}$, TH-GFP ${ }^{+}$neurons taken at E11.5 from the mesencephalon express abundant VGluT2 protein after $24 \mathrm{~h}$ in culture (DIV1), both in the soma and growth cone (white box). Scale bar, $10 \mu \mathrm{m} . \mathbf{G}$, Vglut2 transcript is still present in the mesencephalon at E14.5 and only partly overlaps with $T$ transcript in medial sections of the mesencephalon, but not in lateral sections. Scale bar, $25 \mu \mathrm{m}$.

no difference in the number of DA neurons in the mesencephalon of VGluT2KO E18.5 embryos (Fig. 3B; Student's $t$ test, $p>0.05$ ), demonstrating that embryonic VGluT2 expression in DA neurons is not directly required for DA neuronal cell survival.

Early expression of VGluT2 in the growth cone of DA neurons during embryonic development (Fig. 2) is compatible with a potential role of glutamate release in the axonal development and connectivity of DA neurons. Moreover, we and others observed a role for VGluT2 and glutamate in axonal growth (Schmitz et al., 2009; Fortin et al., 2012). Therefore, we investigated whether the axonal projections of DA neurons in the striatum were altered in the absence of VGluT2 by quantifying the density of TH immunoreactivity in different sectors of the striatum. We observed no overt macroscopic changes in the development of DA striatal innervation in VGluT2KO mice compared with VGluT2WT littermate controls (Fig. 3C). However, we observed a significant decrease in TH-IR in the caudal dorsal striatum (Fig. $3 D$; Student's $t$ test, $p=0.008$ ), but not in the ventral striatum (Fig. $3 E$; Student's $t$ test, $p>0.05$ ), to which VTA DA neurons project. The regional specificity of this decrease in striatal TH-IR should likely be interpreted in the context of the fact that the caudal dorsal striatum is the primordial region that corresponds to the adult target regions of VGluT2-expressing SN DA neurons (Poulin et al., 2018).

To verify whether the regional decrease of TH-IR results from a reduced density of DA axons or from the regional downregulation of $\mathrm{TH}$ protein, we investigated the axonal expression of other markers of DA axons including DAT and VMAT2 on adjacent slides. While hardly any DAT protein could be detected in the striatum at this embryonic stage, in line with previous work (Coulter et al., 1997; Galineau et al., 2004), VMAT2 protein was modestly but clearly detectable at E18.5 in the striatum (Fig. $3 F$ ). Investigation of the DA projection regions in the ventral and dorsal striatum revealed no significant changes in VMAT2IR in the absence of VGluT2 (Student's $t$ test, $p>0.05$; Fig. $3 G$, $H$ ). These results suggest that most of the development of striatal innervation by DA neurons does not depend on the presence of VGluT2 in DA neurons. However, in a small subset of DA neurons that project into the caudal dorsal striatum, the expression of $\mathrm{TH}$ protein is downregulated in the absence of VGluT2. Finally, since such a regional deficit was not described in the conditional VGluT2KO mice (using the Dat promoter as a Cre-driver; Hnasko et al., 2010; Fortin et al., 2012), we propose that such regulation of TH may have been undetectable in conditional $\mathrm{KO}$ mice because it implicates events occurring before E14.5.

\section{VGluT2 promotes axonal growth in cultured DA neurons}

To better understand the contribution of VGluT2 to the development of DA axons, we investigated whether moderate overexpression of VGluT2 enhances the axonal arbor size of primary postnatal DA neurons using a lentiviral VGluT2-Venus construct driven by a synapsin promoter (Fig. 4A). Quantification of VGluT2 immunoreactivity in the axons of these primary DA neurons confirmed that this approach increased the levels of VGluT2 protein by $\sim 50 \%$ (Fig. $4 B$ ), thus staying within a physiological range of expression. Next, using confocal microscopy and MAP2 immunostaining to distinguish between axons $\left(\mathrm{MAP}^{-}\right)$and dendrites $\left(\mathrm{MAP}^{+}\right)$, we reconstructed the complete axonal arborization of these DA neurons (Fig. 4C,D). DA neurons overexpressing VGluT2-Venus displayed a significantly larger axonal arbor compared with control DA neurons transfected with empty Venus vector (Fig. 4E; 6672 vs 9423 pixels, Student's $t$ test, $p<0.004)$. Moreover, the axonal arborization of DA neurons expressing VGluT2-Venus was more complex, based on the increased number of branches (Fig. 4F; Student's $t$ test, $p<0.003$ ), as well as higher scores in a Scholl analysis (Fig. $4 G$; Student's $t$ test, $p=0.04$ ). Interestingly, dendritic size, shape, and complexity were not affected by the overexpression of VGluT2-Venus (Fig. 4H; Student's $t$ test, $p>0.05$ ). Finally, to test whether the overexpression of VGluT2 had effects on DA 

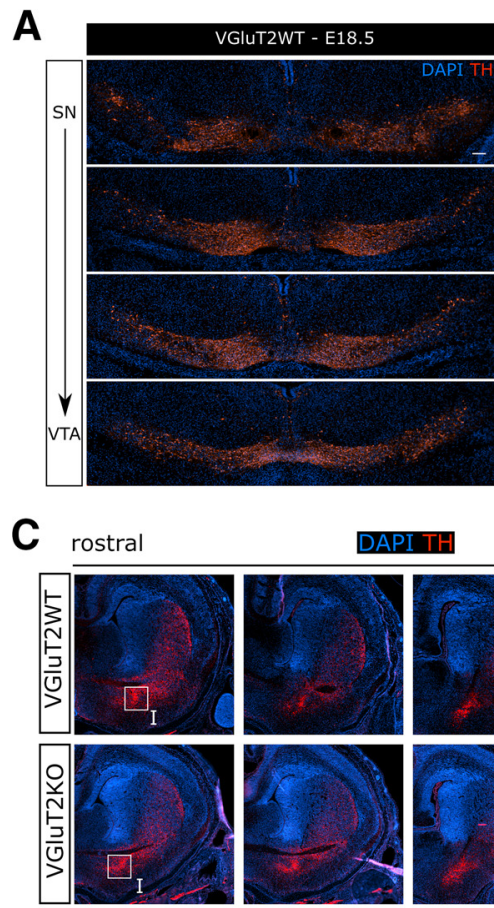

F rostral

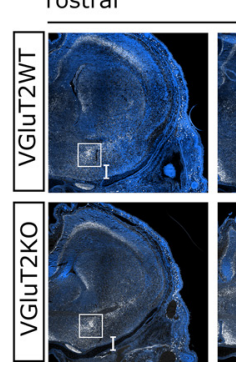

DAPI TH
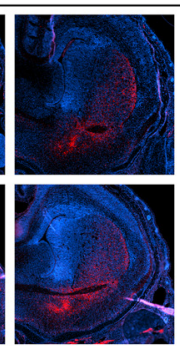

DAPI VMAT2

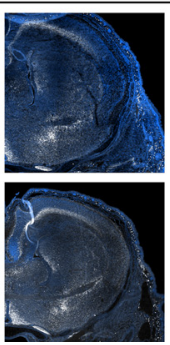

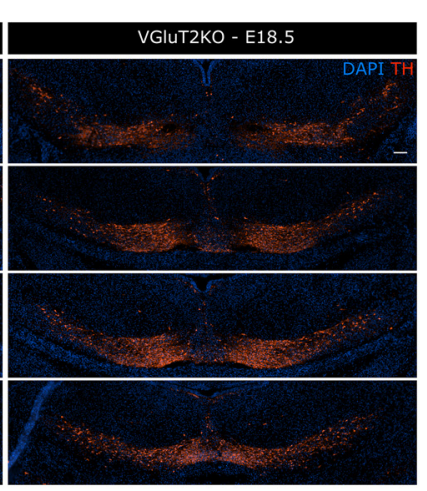
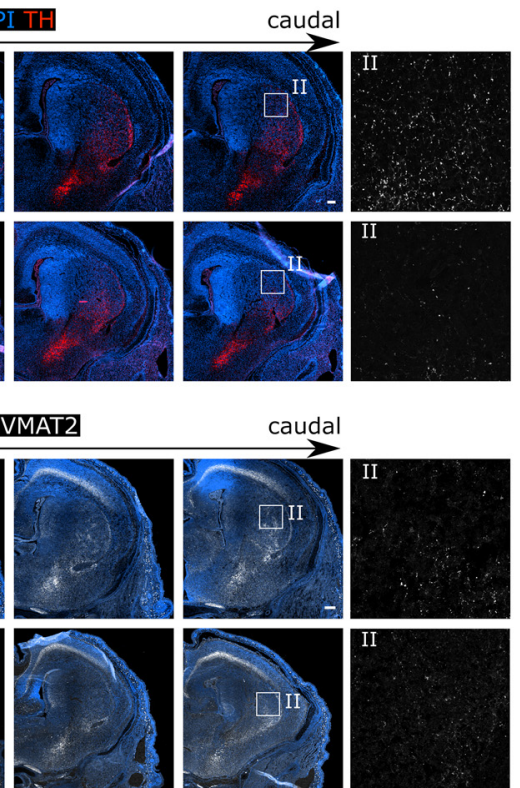
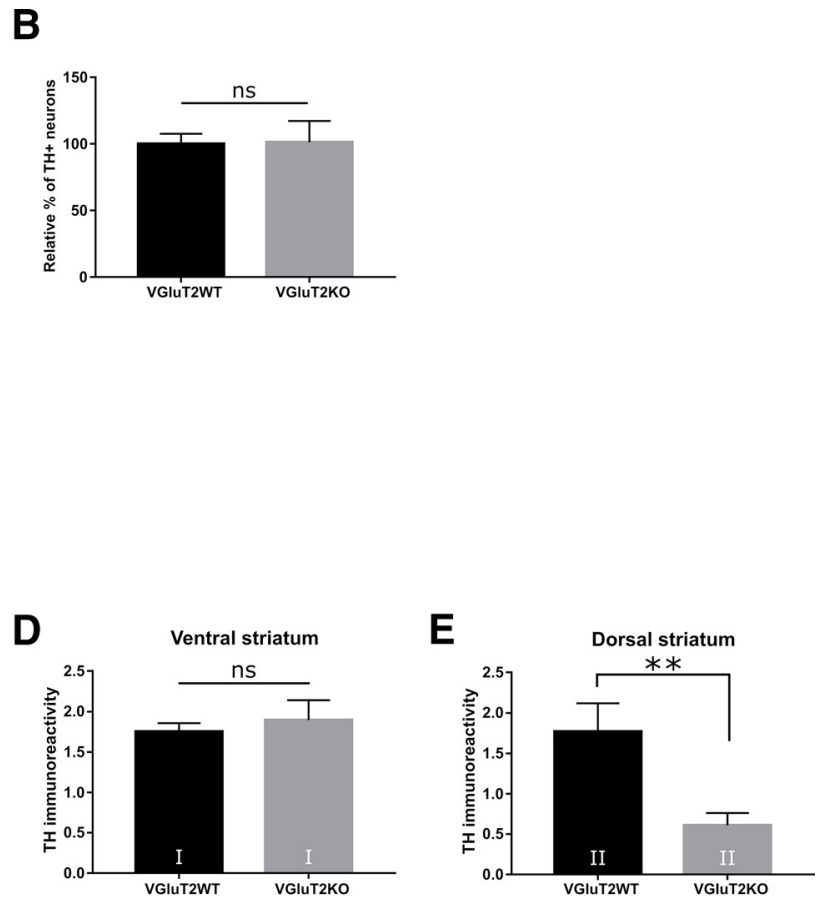

Figure 3. Unaltered DA neuronal development in the absence of VGluT2, but regional striatal innervation deficit. $\boldsymbol{A}$, Immunohistochemistry analysis against TH and DAPI in coronal slices of E18.5 VGluT2WT and constitutive VGluT2KO littermates reveals no macroscopic changes in the mesencephalic dopaminergic system. Scale bar, $100 \mu$ m. $\boldsymbol{B}$, Quantification of the number of TH ${ }^{+}$neurons at E18.5 in the mesencephalon reveals no significant differences between VGluT2WT and VGluT2KO littermates. C, Immunohistochemistry analysis against TH and DAPI in coronal slices of E18.5 VGluT2WT and VGluT2KO littermates reveals no macroscopic changes in the striatum. Scale bar, $100 \mu \mathrm{m}$. $\boldsymbol{D}, \boldsymbol{E}$, Quantification of TH-immunoreactivity in the striatum at E18.5 reveals a regional decrease in intensity in caudal, dorsal striatum ( $\boldsymbol{D}: n=4$ pups, $p<0.01$, Student's $t$ test) but not in ventral striatum ( $\boldsymbol{E}: n=4$ pups, $p>0.05$, Student's $t$ test). ${ }^{* *} p<0.01$. $\boldsymbol{F}$ - $\boldsymbol{H}$, VMAT2 protein is present in the striatum at E18.5. Quantification of VMAT2-immunoreactivity in the ventral and dorsal striatum reveals no significant differences between VGluT2KO mice and their littermate controls $(n=4$ pups, $p>0.05$, Student's $t$ test). ns, no significant difference. Scale bar, $100 \mu \mathrm{m}$.

neuronal survival, we quantified the number of DA neurons per culture and observed no difference in survival between DA neurons overexpressing VGluT2-Venus compared with Venusexpressing DA neurons (Fig. 4I; Student's $t$ test, $p>0.05$ ). We conclude that VGluT2 expression in postnatal DA neurons can contribute to axonal outgrowth.

Another agent known to boost axon outgrowth and complexity in ventral midbrain DA neurons or fetal SN grafts, is GDNF (Widmer et al., 2000; Schaller et al., 2005; Redmond et al., 2009). Interestingly, GDNF expression is augmented in the postlesional striatum (Hidalgo-Figueroa et al., 2012). To test whether GDNF could drive enhanced Vglut2 expression in DA neurons, we treated cultured DA neurons for $3 \mathrm{~h}$ at DIV7 with $3 \mathrm{~nm}$ GDNF. In accordance with this hypothesis, using single-cell real-time PCR, we found that the proportion of DA neurons containing Vglut 2 mRNA increased after a 3 h treatment with 3 nM GDNF, from $45 \%$ of the TH-GFP neurons in control conditions to $77 \%$ after GDNF (Fig. 4J; Fisher's exact test, $p=0.001$ ).

Thus, we conclude that although VGluT2 is not directly involved in DA neuron cell survival, one of its roles in these neurons may be to contribute to the development and connectivity of their axonal arbors. Moreover, our in vitro data show that growth factors like GDNF that are increased after lesions to the DA system can elevate Vglut2 expression in DA neurons.

Fewer striatal-mesencephalic projections after a partial lesion in absence of VGluT2

Based on our finding of elevated expression of Vglut2 in the majority of SN DA neurons in response to cellular stress, and our results showing increased axon development following moderate overexpression of VGluT2, we hypothesized that the increased expression of VGluT2 in DA neurons after a lesion contributes to postlesional compensatory axonal outgrowth of these neurons, promoting reinnervation of the striatum. To test this hypothesis, we performed an intrastriatal 6-OHDA injection to partially lesion the mesencephalon of adult conditional VGluT2cKO mice and controls, as per a previously published protocol (Giguère et al., 2019), and examined the innervation pattern of SN and VTA DA neurons after injection of retrogradely transported fluorescent beads $(\mathrm{Rb})$ in the striatum (Fig. $5 A$ ). We examined the mice 
A

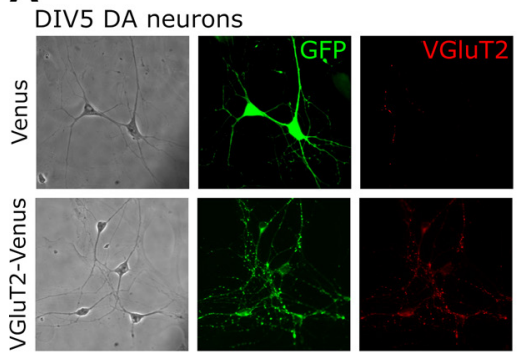

B

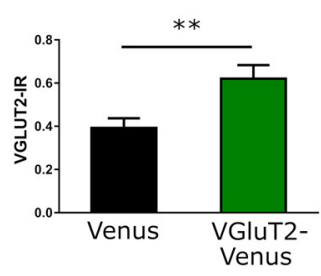

C

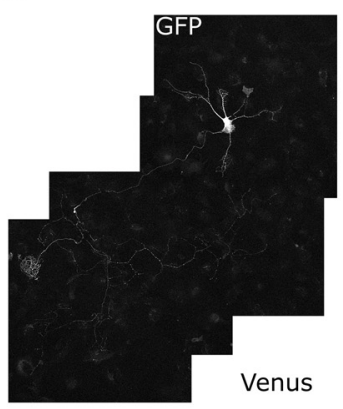

D

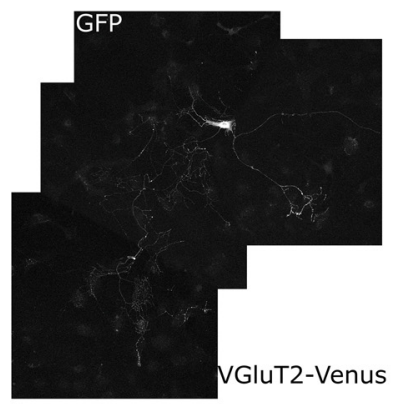

E

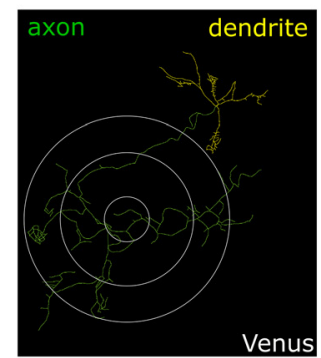

1

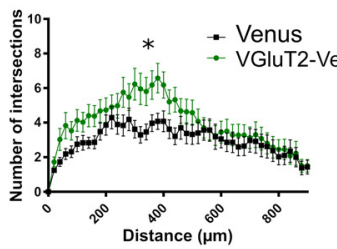

$\mathbf{F}$ axon

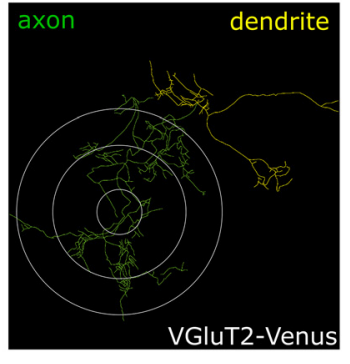

$\mathbf{J}$

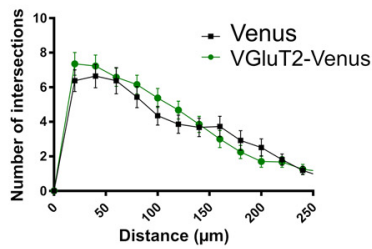

G

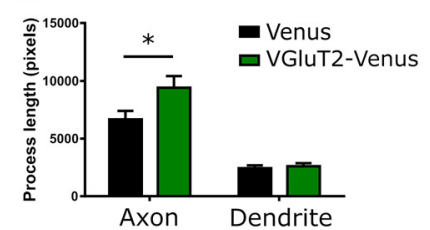

K

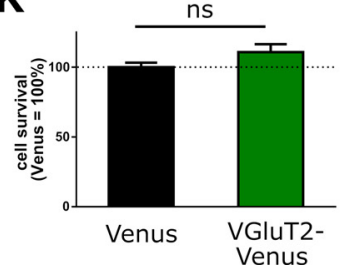

H

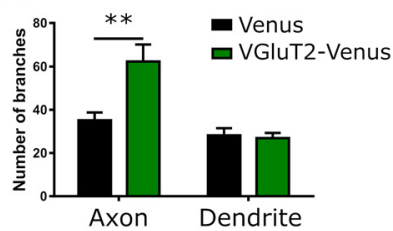

L TH-GFP+ cells

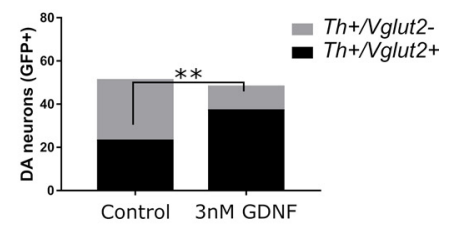

Figure 4. VGluT2 promotes axonal growth in DA neurons in vitro. $\boldsymbol{A}$, TH-GFP ${ }^{+}$mesencephalic neurons were cultured at P1 for $5 \mathrm{~d}$ (DIV5) and infected with VGluT2-Venus lentivirus or control Venus lentivirus. VGluT2 protein is detectable in VGluT2-Venus-infected neurons. B, Quantification of VGluT2 protein levels present in axons revealed a $50 \%$ increase in immunoreactivity ( $n=53-54, p<0.01$, Student's $t$ test). $\mathbf{C}-\boldsymbol{F}$, Mesencephalic neurons overexpressing VGluT2-Venus display a larger axonal arborization and increased number of branches. $\boldsymbol{C}, \boldsymbol{D}$, GFP protein expression was used to visualize the general morphology of the cells. $\boldsymbol{E}, \boldsymbol{F}$, Traced examples of DA neurons, in which the axonal arbor is indicated in green and the dendritic arbor in yellow. The white circles provide visual aid to observe the relative size of the axonal arbor. $\mathbf{G}$, Quantification of the axonal arbor reveals an enhanced arbor in neurons overexpressing VGluT2-Venus ( $n=37-40$ neurons, Student's $t$ test, $p<0.05)$. $\boldsymbol{H}$, Branching analysis shows enhanced complexity in axonal arborization because of VGluT2-Venus overexpression $(n=37-40$ neurons, Student's $t$ test, $p<0.01$ ). I, Viral overexpression of VGluT2-Venus induces increased number of intersections of axons from DA neurons, compared with Venus control (two-way ANOVA on Scholl analysis, $\left.p<0.05, F_{(1,3450)}=57.9\right)$. J, Viral overexpression of VGluT2-Venus did not change the number of intersections of dendrites from DA neurons, compared with Venus control (two-way ANOVA on Scholl analysis, $p>0.05$ ). $K$, Overexpression of VGluT2-Venus did not alter the survival of purified TH-GFP ${ }^{+}$SN DA neurons (Student's $t$ test, $p>0.05$ ). $L$, Cultured DA neurons that were treated for $3 \mathrm{~h}$ with GDNF (3 nm) before being collected were significantly more likely to contain both Vglut2 and Th transcripts compared with DA neurons in untreated cultured ( $n=40$ cells, four cultures, Fisher's exact test, $p<0.01$ ). ${ }^{*} p<0.05,{ }^{* *} p<0.01$, ns, no significant difference.

7 weeks after the lesion because postlesional compensatory reinnervation of the striatum has been observed at this time following 6-OHDA injection (Schmitz et al., 2013). We hypothesized that in the absence of VGluT2, DA neurons would be less successful in reinnervating the striatum compared with wild-type controls (Fig. 5A). To quantify the reinnervation, we injected red fluorescent $\mathrm{Rb}$ at the same coordinates as the 6-OHDA or saline injection (AP, $0.5 \mathrm{~mm}$; ML, $2.0 \mathrm{~mm}$; DV, $-3.0 \mathrm{~mm}$ ), 1 week before animals were killed. $\mathrm{Rb}$ were taken up by terminals and transported to the soma, revealing which midbrain neurons project to the dorsolateral striatum at the 7 week postlesion time point. Only brains displaying successful lesions (as determined by clear TH-IR loss within the striatal lesion) were included in the analysis, whereas brains displaying mislocated $\mathrm{Rb}$ injections (i.e., outside the striatal lesion zone) were excluded from analysis (Fig. $5 A$, insert). $\mathrm{Rb}^{+}$neurons were found in both the $\mathrm{SN}$ and VTA regions, mainly colocalizing with $\mathrm{TH}^{+}$neurons (Fig. $5 B$, yellow arrow), as expected. We nonetheless detected a limited number of labeled neurons that appeared to be $\mathrm{TH}^{-}$(Fig. $5 \mathrm{~B}$, yellow arrowhead). We observed no significant change in the percentage of $\mathrm{Rb}^{+} / \mathrm{TH}^{-}$cells (compared with total $\mathrm{Rb}^{+}$cells) between conditions (data not shown). Using unbiased stereological counting on TH-stained slices, we observed a $48 \%$ decrease of the number of $\mathrm{TH}^{+} \mathrm{SN}$ neurons in controls and a $44 \%$ decrease in VGluT2cKO animals (Fig. $5 C ; p=0.015, F_{(1,25)}=6.8, n=6-9$ animals/condition), thus confirming that the injection of $1.5 \mu \mathrm{g}$ of 6-OHDA $(0.5 \mu \mathrm{l}, 3 \mathrm{mg} / \mathrm{ml})$ successfully resulted in a partial SN lesion.

Next, to assess the success with which striatal-mesencephalic projections had been re-established in the postlesional brain in the absence of VGluT2, we quantified the total number of $\mathrm{Rb}^{+}$ neurons as well as $\mathrm{Rb}^{+} / \mathrm{TH}^{+}$neurons in the mesencephalon. To compensate for biased quantification because of variations in injection volume, we divided the number of counted $\mathrm{Rb}^{+}$cells by the total surface area of the striatal $\mathrm{Rb}$ injection site. This revealed that in VGluT2cWT control mice, the 6-OHDA lesion caused a modest but nonsignificant decrease in the number of labeled DA neurons, suggesting the presence of efficient compensatory axonal sprouting (Fig. 5D: total $\mathrm{Rb}^{+}$neurons $F_{(1,20)}=12.28$, post hoc $p<0.05$; Fig. $5 E: \mathrm{TH}^{+} / \mathrm{Rb}^{+}$neurons, $F_{(1,19)}=15.44$, post hoc $\left.p>0.05\right)$. In contrast, in VGluT2cKO mice, the 6-OHDA lesion caused a robust decrease in the number of labeled neurons in mesencephalon (Fig. 5D,E: $F_{(1,20)}=$ 12.28, post hoc $p=0.007)$; Fig. $5 E: F_{(1,19)}=15.44$, post hoc $p=0.006)$, suggesting that fewer striatal-mesencephalic projections were present at this seventh week postlesion time point in 

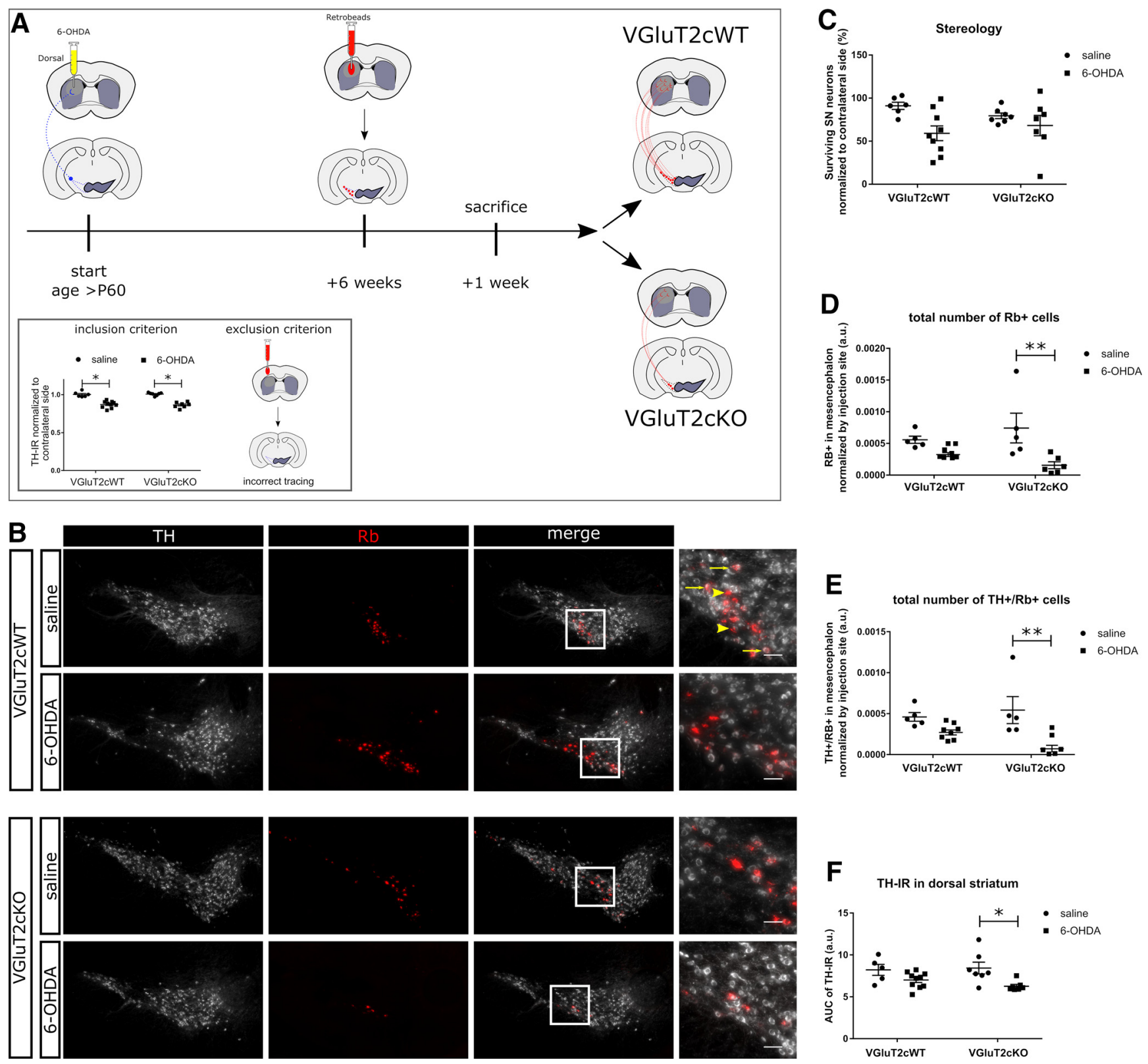

Figure 5. Striatal re-innervation by DA neurons after a partial lesion is perturbed in the absence of VGluT2. A, Schematic detailing of the surgery paradigm and hypothesis that fewer retrobead-positive DA neurons successfully established projections to the dorsal striatum in VGluT2cKO mice after a 6-OHDA lesion compared with wild-type control mice. Inset, Schematic representation of inclusion/exclusion criteria for Rb-tracing study. B, Immunohistochemistry for TH (white signal) reveals the presence of red-fluorescent retrobead-positive cells in the SN, which were typically positive (yellow arrowhead) or more rarely negative for TH-immunoreactivity (yellow arrow). Scale bar, $200 \mu \mathrm{m}$. C, Unbiased stereological counting on sections stained for TH and Cresyl Violet confirmed the loss of SN TH-IR neurons but revealed no increased sensitivity of VGluT2-ablated SN neurons to 6-OHDA (treatment effect: $F_{(1,24)}=6.41, p<0.05, n=5-7$ animals/ condition). $\boldsymbol{D}$, Fewer retrobead-positive neurons are observed in the mesencephalon of VGluT2cKO animals, after 6-OHDA lesion compared with saline controls (treatment effect: $F_{(1,20)}=13.99$, $p<0.01$ ), $n=5-8$ animals/condition. $\boldsymbol{E}$, Fewer $\mathrm{TH}^{+} / \mathrm{RB}^{+}$neurons are observed in the mesencephalon of VGluT2cKO animals, after 6-OHDA lesion compared with saline controls (treatment effect: $\left.F_{(1,20)}=14.92, p<0.01\right), n=5-8$ animals/condition. $F$, Quantification of striatal DAB TH-IR in total lesion per brain confirmed a significant decrease of TH innervation in VGluT2ck0 animals after 6-OHDA injections (treatment effect: $\left.F_{(1,24)}=12.17, p<0.01, n=6-9\right)$. Tukey's multiple-comparison test, ${ }^{*} p<0.05$; ${ }^{* *} p<0.01$.

the VGluT2cKO mice. To validate these results, we also directly quantified the density of dopaminergic fibers reinnervating the striatum using TH-DAB immunohistochemistry, thus avoiding nonspecific fluorescence within the lesioned area caused by the $\mathrm{Rb}$ injection. We quantified the total dorsal striatal lesion size per animal by compiling the intensity of the relative DAB TH-IR per slice of the ipsilesional side compared with the contralateral side and calculating the area under the curve. Compatible with the observation that fewer striatal-mesencephalic projections were present at this 7 week postlesion time point in the VGluT2cKO mice, we observed a decrease in total DAB TH-IR within the dorsal striatum area across both genotypes $\left(F_{(1,25)}=\right.$ $12.43, p<0.01$ ), but post hoc testing revealed a significant decrease only in VGluT2cKO mice (Fig. $5 F ; p=0.02,8.4$ vs 6.3). Of note, because only brains with an evident striatal lesion were included in this analysis (Fig. 6A, inset), the absence of a significant decrease in TH-IR within the total dorsal striatum in the VGluT2cWT mice, is likely to reflect the inclusion of dorsal striatal slices in which TH-IR levels had recovered close to normal levels because of postlesion compensatory plasticity. In summary, our findings suggest that the same number of surviving DA neurons re-established fewer $\mathrm{TH}^{+}$striatal connections in the 
absence of VGluT2, suggesting that VGluT2 plays an important role in driving the growth of striatal-mesencephalic projections in the postlesional brain.

\section{Discussion}

VGluT2 expression in DA neurons could facilitate autocrine glutamate release to drive axonal outgrowth

We previously provided evidence suggesting upregulation of Vglut2 in surviving DA neurons after a neurotoxic lesion (Dal Bo et al., 2008), a finding recently confirmed by two other groups (Shen et al., 2018; Steinkellner et al., 2018). Here, we set out to understand some of the physiological implications of such VGluT2 upregulation. We first used an in vitro approach to quantify the level of Vglut2 expression present in primary neonatal DA neurons. We observed that at birth, $28-45 \%$ of DA neurons express $>20$ or 10 copies of Vglut2, respectively. After $\mathrm{MPP}^{+}$exposure, we observed a 2.5fold increase of Vglut2 mRNA levels in DA neurons, which increased the proportion of Vglut2 $^{+}$DA neurons to $60 \%$ (Fig. 1, Table 1). Importantly, we confirmed previous reports that during embryonic development most DA neurons express VGluT2, while only a minority of the DA neurons maintains this VGluT2 ${ }^{+}$identity in the adult brain (for a review systematically classifying several single-cell profiling papers on DA subsets, see Poulin et al., 2020). This underlines the exceptional plasticity of VGluT2 expression in DA neurons that is observed during the transition from the developing brain to the adult brain as well as during the transition from the healthy adult brain to the postlesional brain (Figs. 1, 2, Table 1). Notably, using a 10 mRNA copy number criterion, we observed that the proportion of Vglut $^{+}$DA neurons does not significantly change upon $\mathrm{MPP}^{+}$ treatment, suggesting that only DA neurons that already minimally expressed ( $\geq 10$ copies) Vglut 2 were able to enhance their expression of this gene in response to cellular stress (Fig. 6A). Whether all subtypes of SN DA neurons, such as Sox6 ${ }^{+}$or $\mathrm{Ndnf}^{+}$SN DA neurons (Poulin et al., 2018, 2020) are equally likely to reactivate abovethreshold expression of Vglut2, or whether SN neuron subsets that are more vulnerable to neurotoxic lesions (e.g., Aldhla1 ${ }^{+}$) are not able to reactivate Vglut2 expression above threshold remains to be determined. Future experiments exposing different intersectional reporter mice (e.g., Th-Flp; Ahdla1-Cre or Th-Flp; Sox6-Cre; Poulin et al., 2018) to neurotoxin lesions are planned to answer these questions.

To understand the physiological consequences of the reactivated VGluT2 expression profile in DA neurons, we mimicked this change in primary neonatal DA neurons and found that moderate overexpression of VGluT2 protein resulted in a larger and more complex axonal arborization (Fig. 4), complementing the observation that DA neurons grow smaller axonal arbori- zations in the absence of VGluT2 (Fortin et al., 2012). Finally, when we exposed mice lacking VGluT2 in DA neurons to a partial lesion, we observed less compensatory striatal sprouting than in control animals (Fig. 5).

Previously published work suggested that an autocrine feedback loop of glutamate release by primary DA neurons drives axonal outgrowth, mediated by the ionotropic NMDA glutamate receptor subunit NR1 found on the growth cone of DA neurons (Schmitz et al., 2009). Here we propose the following model integrating these two observations: VGluT2 expression in growth cones of primary DA neurons allows the release of glutamate, which will stimulate glutamate receptors on DA neurons and promote axonal outgrowth (Fig. 6B). Moreover, we propose that the reactivation of VGluT2 expression in surviving DA neurons that occurs in the postlesional brain could potentially reactivate the autocrine glutamate feedback loop. Consequently, DA axonal outgrowth can be stimulated, which could contribute to compensatory sprouting after a lesion (Fig. 6C). We are not proposing here that such an autocrine glutamate feedback loop is the sole mechanisms regulating axonal outgrowth in primary DA neurons or in DA neurons in the postlesional brain. Indeed, the 
effect size of VGluT2 overexpression or VGluT2 ablation on axonal arborization is relatively modest (i.e., $\sim 15-30 \%$ in both the gain-of-function and loss-of-function study (Fig. 4; Fortin et al., 2012). In addition, compensatory sprouting after a lesion to DA neurons was not completely abolished in VGluT2cKO mice (Fig. 5). A recent study showing that several axonal guidance factors are decreased in the postlesional brain (Kalaani et al., 2016) underscores the possibility that multiple mechanisms contribute to suboptimal compensatory axonal outgrowth after lesions.

The mechanism by which VGluT2 is upregulated in DA neurons in response to toxic stress is presently unknown. However, we previously reported that cultured DA neurons are less likely to express VGluT2 in the presence of dorsal striatal cells than DA neurons cultured by themselves or in the presence of ventral striatal cells (Fortin et al., 2019), suggesting that dorsal striatal cells might exert a repressive effect on VGluT2 expression in DA neurons. Notably, in the context of a neurotoxic treatment, the connections between DA neurons and (dorsal) striatum will be lesioned, and as such a potential inhibitory striatal signal would be lifted and permit reactivation of VGluT2 expression in DA neurons. In line with this observation, excitotoxic lesioning of striatal neurons also elevates Vglut2 expression in DA neurons (Mendez et al., 2008). Second, we demonstrated here that GDNF promotes the double Vglut2/Th phenotype in cultured DA neurons, in line with our previous work showing that GDNF promotes glutamate release by primary DA neurons (Bourque and Trudeau, 2000). It is also worth noting that GDNF expression is augmented in the striatum after lesions (Hidalgo-Figueroa et al., 2012) and is known to promote neurite outgrowth and complexity of DA neurons in cultures (Widmer et al., 2000; Schaller et al., 2005) as well as DA cell replacement grafts (Redmond et al., 2009; Kauhausen et al., 2013).

\section{Effect of VGluT2 expression on survival and toxicity}

A growing body of work now suggests that VGluT2 expression may promote the survival of DA neurons. Following our initial work performed using an early postnatal lesion model (Dal Bo et al., 2008), two other groups recently tested this hypothesis. First, using neurotoxic lesions, both groups found larger lesions in the VGluT2cKO mice compared with the VGluT2cWT mice, suggesting that the expression of VGluT2 renders cells more resistant to the insult (Shen et al., 2018; Steinkellner et al., 2018). In this context, it may be surprising to note that in the present work, we did not observe enhanced loss of DA neurons in the VGluT2cKO mice after a 6-OHDA lesion, although we did observe decreased dopaminergic innervation of the striatum in VGluT2cKO animals after the lesion (Fig. 5). Compared with the work of Steinkellner et al. (2018), who also used 6-OHDA lesions, we noticed the following two main experimental differences that should be considered: first, to induce a partial lesion, we used a dose of 6-OHDA 50\% lower than that used by these authors. Second, to investigate compensatory reinnervation of the striatum by DA neurons, we collected the brains 2 months postlesion, compared with $10 \mathrm{~d}$ in their study (Steinkellner et al., 2018). It is well established that the effect of 6-OHDA and other neurotoxins on DA neurons consists of both the downregulation of TH expression and secondary loss of DA neurons. Several studies demonstrated that the time immediately after a lesion is characterized by strong downregulation of TH expression, a cellular phenotype that can partially recover, while genuine cell loss is more apparent at later timepoints (Bowenkamp et al., 1996; Stanic et al., 2003). From that perspective, it could be speculated that DA neurons lacking VGluT2 may downregulate $\mathrm{TH}$ more readily in response to cellular stress compared with control animals, without necessarily progressing more extensively toward cell death.

Second, to test whether VGluT2 expression contributes to DA neuron survival, both groups opted to overexpress VGluT2 in DA neurons in vivo, using an adeno-associated virus (AAV) vector. Intriguingly, while the first published article reported significant cell loss of DA neurons following VGluT2 overexpression (Steinkellner et al., 2018), the second published article reported opposite results: overexpression of VGluT2 was able to prevent neurotoxin-induced DA neuronal loss (Shen et al., 2018). Intriguingly, this discrepancy with previous work was not discussed. In the current manuscript, we used a lentiviral approach to overexpress VGluT2 by only $50 \%$ (Fig. 4). Knowing that in vitro $\mathrm{MPP}^{+}$treatment induced an $\sim 100 \%$ increase of Vglut 2 expression in DA neurons (Fig. 1), we believe our overexpression of VGluT2 within DA neurons stayed well within a physiological range. In contrast, in the previous reports, AAV-mediated gene transfer, a strategy that is known to result in highly efficient gene expression (Saraiva et al., 2016), is likely to have led to supraphysiological elevations of VGluT2 levels in DA neurons. It is thus reasonable to hypothesize that in the study by Steinkellner et al. (2018), such nonphysiological levels of VGluT2 may have caused cell death. Future research quantifying VGluT2 protein levels in healthy DA neurons, as well as after an AAVinduced VGluT2 overexpression, could potentially help to clarify this issue. In the present study, we did not find evidence in support of the data from Shen et al. (2018) proposing that enhanced VGluT2 expression promotes the survival of DA neurons (Fig. 4). In addition, we did not observe any loss of DA neurons in the constitutive VGluT2 KO embryos at E18.5 compared with their wild-type littermate control (Fig. 3). Together, we conclude from our data that physiological VGluT2 expression in DA neurons, although promoting striatal reinnervation, does not directly contribute to DA neuron survival.

\section{Closing remarks}

Previous work showed that an uptake inhibitor of the amino acid glycine, a coagonist of the NMDA glutamate receptor, can promote reinnervation of the dorsal striatum after a 6-OHDA lesion (Schmitz et al., 2013). It was proposed that this mechanism could potentially be used in $\mathrm{PD}$ patients to promote innervation of striatum by surviving DA neurons. The present work adds support to this possibility by demonstrating that VGluT2 expression in DA neurons and thus glutamate release by these neurons may represent an endogenous mechanism acting to facilitate reinnervation of the striatum in parallel with the gradual loss of DA neurons during the course of this disease.

\section{References}

Aguilar JI, Dunn M, Mingote S, Karam CS, Farino ZJ, Sonders MS, Choi SJ, Grygoruk A, Zhang Y, Cela C, Choi BJ, Flores J, Freyberg RJ, McCabe BD, Mosharov EV, Krantz DE, Javitch JA, Sulzer D, Sames D, Rayport S, et al. (2017) Neuronal depolarization drives increased dopamine synaptic vesicle loading via VGLUT. Neuron 95:1074-1088.

Arenas E, Denham M, Villaescusa JC (2015) How to make a midbrain dopaminergic neuron. Development 142:1918-1936. 
Bäckman CM, Malik N, Zhang Y, Shan L, Grinberg A, Hoffer BJ, Westphal H, Tomac AC (2006) Characterization of a mouse strain expressing Cre recombinase from the $3^{\prime}$ untranslated region of the dopamine transporter locus. Genesis 44:383-390.

Bérubé-Carrière N, Guay G, Fortin GM, Kullander K, Olson L, WallénMackenzie Å, Trudeau L-E, Descarries L (2012) Ultrastructural characterization of the mesostriatal dopamine innervation in mice, including two mouse lines of conditional VGLUT2 knockout in dopamine neurons. Eur J Neurosci 35:527-538.

Bourque MJ, Trudeau LE (2000) GDNF enhances the synaptic efficacy of dopaminergic neurons in culture. Eur J Neurosci 12:3172-3180.

Bowenkamp KE, David D, Lapchak PL, Henry MA, Granholm AC, Hoffer BJ, Mahalik TJ (1996) 6-hydroxydopamine induces the loss of the dopaminergic phenotype in substantia nigra neurons of the rat. Exp Brain Res 111:1-7.

Chuhma N, Mingote S, Yetnikoff L, Kalmbach A, Ma T, Ztaou S, Sienna AC, Tepler S, Poulin J-F, Ansorge M, Awatramani R, Kang UJ, Rayport S (2018) Dopamine neuron glutamate cotransmission evokes a delayed excitation in lateral dorsal striatal cholinergic interneurons. eLife 7:e39786.

Cliburn RA, Dunn AR, Stout KA, Hoffman CA, Lohr KM, Bernstein AI, Winokur EJ, Burkett J, Schmitz Y, Caudle WM, Miller GW (2017) Immunochemical localization of vesicular monoamine transporter 2 (VMAT2) in mouse brain. J Chem Neuroanat 83-84:82-90.

Coulter CL, Happe HK, Murrin LC (1997) Dopamine transporter development in postnatal rat striatum: an autoradiographic study with [3H]WIN 35,428. Brain Res Dev Brain Res 104:55-62.

Dal Bo G, Bérubé-Carrière N, Mendez JA, Leo D, Riad M, Descarries L, Lévesque D, Trudeau L-E (2008) Enhanced glutamatergic phenotype of mesencephalic dopamine neurons after neonatal 6-hydroxydopamine lesion. Neuroscience 156:59-70.

Dumas S, Wallén-Mackenzie $\AA$ (2019) Developmental co-expression of Vglut2 and Nurr1 in a Mes-Di-encephalic continuum precedes dopamine and glutamate neuron specification. Front Cell Dev Biol 7:307.

Fasano C, Thibault D, Trudeau L-E (2008) Culture of postnatal mesencephalic dopamine neurons on an astrocyte monolayer. Curr Protoc Neurosci 3:Unit 3.21.

Fasano C, Rocchetti J, Pietrajtis K, Zander JF, Manseau F, Sakae DY, MarcusSells M, Ramet L, Morel LJ, Carrel D, Dumas S, Bolte S, Bernard V, Vigneault E, Goutagny R, Ahnert-Hilger G, Giros B, Daumas S, Williams S, El Mestikawy S (2017) Regulation of the Hippocampal Network by VGLUT3-Positive CCK- GABAergic Basket Cells. Front Cell Neurosci 11:140.

Fortin GM, Bourque M-J, Mendez JA, Leo D, Nordenankar K, Birgner C, Arvidsson E, Rymar VV, Bérubé-Carrière N, Claveau A-M, Descarries L, Sadikot AF, Wallén-Mackenzie Å, Trudeau L-É (2012) Glutamate corelease promotes growth and survival of midbrain dopamine neurons. J Neurosci 32:17477-17491.

Fortin GM, Ducrot C, Giguère N, Kouwenhoven WM, Bourque M-J, Pacelli C, Varaschin RK, Brill M, Singh S, Wiseman PW, Trudeau L-E (2019) Segregation of dopamine and glutamate release sites in dopamine neuron axons: regulation by striatal target cells. FASEB J 33:400-417.

Galineau L, Kodas E, Guilloteau D, Vilar M-P, Chalon S (2004) Ontogeny of the dopamine and serotonin transporters in the rat brain: an autoradiographic study. Neurosci Lett 363:266-271.

Giguère N, Delignat-Lavaud B, Herborg F, Voisin A, Li Y, Jacquemet V, Anand-Srivastava M, Gether U, Giros B, Trudeau L-É (2019) Increased vulnerability of nigral dopamine neurons after expansion of their axonal arborization size through D2 dopamine receptor conditional knockout. PLoS Genet 15:e1008352.

Gras C, Amilhon B, Lepicard EM, Poirel O, Vinatier J, Herbin M, Dumas S, Tzavara ET, Wade MR, Nomikos GG, Hanoun N, Saurini F, Kemel M-L, Gasnier B, Giros B, El Mestikawy S (2008) The vesicular glutamate transporter VGLUT3 synergizes striatal acetylcholine tone. Nat Neurosci 11:292-300.

Grima B, Lamouroux A, Blanot F, Biguet NF, Mallet J (1985) Complete coding sequence of rat tyrosine hydroxylase mRNA. Proc Natl Acad Sci USA $82: 617-621$

Herzog E, Bellenchi GC, Gras C, Bernard V, Ravassard P, Bedet C, Gasnier B, Giros B, El Mestikawy S (2001) The existence of a second vesicular glutamate transporter specifies subpopulations of glutamatergic neurons. J Neurosci 21:RC181
Herzog E, Nadrigny F, Silm K, Biesemann C, Helling I, Bersot T, Steffens H, Schwartzmann R, Nägerl UV, El Mestikawy S, Rhee J, Kirchhoff F, Brose N (2011) In vivo imaging of intersynaptic vesicle exchange using VGLUT1 Venus knock-in mice. J Neurosci 31:15544-15559.

Hidalgo-Figueroa M, Bonilla S, Gutiérrez F, Pascual A, López-Barneo J (2012) GDNF is predominantly expressed in the $\mathrm{PV}^{+}$neostriatal interneuronal ensemble in normal mouse and after injury of the nigrostriatal pathway. J Neurosci 32:864-872.

Hnasko TS, Chuhma N, Zhang H, Goh GY, Sulzer D, Palmiter RD, Rayport S, Edwards RH (2010) Vesicular glutamate transport promotes dopamine storage and glutamate corelease in vivo. Neuron 65:643-656.

Jacobs FMJ, Smits SM, Noorlander CW, von Oerthel L, van der Linden AJA, Burbach JPH, Smidt MP (2007) Retinoic acid counteracts developmental defects in the substantia nigra caused by Pitx3 deficiency. Dev Camb Engl 134:2673-2684.

Kalaani J, Roche J, Hamade E, Badran B, Jaber M, Gaillard A, Prestoz L (2016) Axon guidance molecule expression after cell therapy in a mouse model of Parkinson's disease. Restor Neurol Neurosci 34:877-895.

Kauhausen J, Thompson LH, Parish CL (2013) Cell intrinsic and extrinsic factors contribute to enhance neural circuit reconstruction following transplantation in Parkinsonian mice. J Physiol 591:77-91.

Li X, Qi J, Yamaguchi T, Wang H-L, Morales M (2013) Heterogeneous composition of dopamine neurons of the rat A10 region: molecular evidence for diverse signaling properties. Brain Struct Funct 218:1159-1176.

Matsushita N, Okada H, Yasoshima Y, Takahashi K, Kiuchi K, Kobayashi K (2002) Dynamics of tyrosine hydroxylase promoter activity during midbrain dopaminergic neuron development. J Neurochem 82:295-304.

Mendez JA, Bourque M-J, Dal Bo G, Bourdeau ML, Danik M, Williams S, Lacaille J-C, Trudeau L-E (2008) Developmental and target-dependent regulation of vesicular glutamate transporter expression by dopamine neurons. J Neurosci 28:6309-6318.

Moechars D, Weston MC, Leo S, Callaerts-Vegh Z, Goris I, Daneels G, Buist A, Cik M, van der Spek P, Kass S, Meert T, D’Hooge R, Rosenmund C, Hampson RM (2006) Vesicular glutamate transporter VGLUT2 expression levels control quantal size and neuropathic pain. J Neurosci 26:12055-12066.

Poulin J-F, Caronia G, Hofer C, Cui Q, Helm B, Ramakrishnan C, Chan CS, Dombeck DA, Deisseroth K, Awatramani R (2018) Mapping projections of molecularly defined dopamine neuron subtypes using intersectional genetic approaches. Nat Neurosci 21:1260-1271.

Poulin J-F, Gaertner Z, Moreno-Ramos OA, Awatramani R (2020) Classification of midbrain dopamine neurons using single-cell gene expression profiling approaches. Trends Neurosci 43:155-169.

Redmond DE, Elsworth JD, Roth RH, Leranth C, Collier TJ, Blanchard B, Bjugstad KB, Samulski RJ, Aebischer P, Sladek JR (2009) Embryonic substantia nigra grafts in the mesencephalon send neurites to the host striatum in non-human primate after overexpression of GDNF. J Comp Neurol 515:31-40.

Saraiva J, Nobre RJ, Pereira de Almeida L (2016) Gene therapy for the CNS using AAVs: the impact of systemic delivery by AAV9. J Control Release 241:94-109.

Schaller B, Andres RH, Huber AW, Meyer M, Pérez-Bouza A, Ducray AD, Seiler RW, Widmer HR (2005) Effect of GDNF on differentiation of cultured ventral mesencephalic dopaminergic and non-dopaminergic calretinin-expressing neurons. Brain Res 1036:163-172.

Schmitz Y, Luccarelli J, Kim M, Wang M, Sulzer D (2009) Glutamate controls growth rate and branching of dopaminergic axons. J Neurosci 29:1197311981.

Schmitz Y, Castagna C, Mrejeru A, Lizardi-Ortiz JE, Klein Z, Lindsley CW, Sulzer D (2013) Glycine transporter-1 inhibition promotes striatal axon sprouting via NMDA receptors in dopamine neurons. J Neurosci 33:16778-16789.

Shen H, Marino RAM, McDevitt RA, Bi G-H, Chen K, Madeo G, Lee P-T, Liang Y, De Biase LM, Su T-P, Xi Z-X, Bonci A (2018) Genetic deletion of vesicular glutamate transporter in dopamine neurons increases vulnerability to MPTP-induced neurotoxicity in mice. Proc Natl Acad Sci U S A 115:E11532-E11541.

Silm K, Yang J, Marcott PF, Asensio CS, Eriksen J, Guthrie DA, Newman AH, Ford CP, Edwards RH (2019) Synaptic vesicle recycling pathway determines neurotransmitter content and release properties. Neuron 102:786-800.e5. 
Smidt MP, Burbach JPH (2007) How to make a mesodiencephalic dopaminergic neuron. Nat Rev Neurosci 8:21-32.

Stanic D, Finkelstein DI, Bourke DW, Drago J, Horne MK (2003) Timecourse of striatal re-innervation following lesions of dopaminergic SNpc neurons of the rat. Eur J Neurosci 18:1175-1188.

Steinkellner T, Zell V, Farino ZJ, Sonders MS, Villeneuve M, Freyberg RJ, Przedborski S, Lu W, Freyberg Z, Hnasko TS (2018) Role for VGLUT2 in selective vulnerability of midbrain dopamine neurons. J Clin Invest 128:774-788.

Wallén-Mackenzie A, Gezelius H, Thoby-Brisson M, Nygård A, Enjin A, Fujiyama F, Fortin G, Kullander K (2006) Vesicular glutamate transporter 2 is required for central respiratory rhythm generation but not for locomotor central pattern generation. J Neurosci 26:12294-12307.

Widmer HR, Schaller B, Meyer M, Seiler RW (2000) Glial cell line-derived neurotrophic factor stimulates the morphological differentiation of cultured ventral mesencephalic calbindin- and calretinin-expressing neurons. Exp Neurol 164:71-81.

Yamaguchi T, Sheen W, Morales M (2007) Glutamatergic neurons are present in the rat ventral tegmental area. Eur J Neurosci 25:106118.

Yamaguchi T, Wang H-L, Morales M (2013) Glutamate neurons in the substantia nigra compacta and retrorubral field. Eur J Neurosci 38:36023610.

Yamaguchi T, Qi J, Wang H-L, Zhang S, Morales M (2015) Glutamatergic and dopaminergic neurons in the mouse ventral tegmental area. Eur J Neurosci 41:760-772.

Zhang S, Qi J, Li X, Wang H-L, Britt JP, Hoffman AF, Bonci A, Lupica CR, Morales M (2015) Dopaminergic and glutamatergic microdomains in a subset of rodent mesoaccumbens axons. Nat Neurosci 18:386-392. 